University of South Florida

DIGITAL COMMONS

Digital Commons @ University of

@ UNIVERSITY OF SOUTH FLORIDA

South Florida

USF Accountability Reports

USF Archives

$1-1-2011$

\title{
2011 Work Plan USF System
}

USF

Follow this and additional works at: https://digitalcommons.usf.edu/usf_accountability_reports

\section{Scholar Commons Citation}

USF, "2011 Work Plan USF System" (2011). USF Accountability Reports. 24.

https://digitalcommons.usf.edu/usf_accountability_reports/24

This Article is brought to you for free and open access by the USF Archives at Digital Commons @ University of South Florida. It has been accepted for inclusion in USF Accountability Reports by an authorized administrator of Digital Commons @ University of South Florida. For more information, please contact digitalcommons@usf.edu. 


\section{Update to the \\ University of South Florida System \\ Work Plan \\ Pending BOT Approval}


Note concerning data accuracy: The Office of the Board of Governors believes that the accuracy of the data it collects and reports is paramount to ensuring accountability in the State University System. Thus, the Board Office allows university resubmissions of some data to correct errors when they are discovered. This policy can lead to changes in historical data. 


\begin{tabular}{|c|c|c|c|c|c|c|c|}
\hline \multicolumn{8}{|c|}{ University of South Florida 2010 Annual Report } \\
\hline \multicolumn{3}{|c|}{ Sites and Campuses } & \multicolumn{5}{|c|}{ USF Tampa Campus, USF St. Petersburg Campus, USF Sarasota-Manatee Campus, USF Polytechnic Campus } \\
\hline Enrollments & Headcount & $\%$ & \multicolumn{3}{|c|}{ Degree Programs Offered (As of Spr. 10) } & \multicolumn{2}{|c|}{ Carnegie Classification } \\
\hline $\begin{array}{l}\text { TOTAL } \\
\text { (Fall 2009) }\end{array}$ & 47,306 & $100 \%$ & \multicolumn{2}{|l|}{ TOTAL } & 233 & $\begin{array}{c}\text { Undergraduate } \\
\text { Instructional Program: }\end{array}$ & $\begin{array}{l}\text { Balanced arts \& sciences/professions, } \\
\text { high graduate coexistence }\end{array}$ \\
\hline Black & 5,284 & $11 \%$ & \multicolumn{2}{|c|}{ Baccalaureate } & 92 & Graduate Instructional & Comprehensive doctoral \\
\hline Hispanic & 6,242 & $13 \%$ & \multicolumn{2}{|c|}{ Master's \& Specialist's } & 100 & Program: & with medical/veterinary \\
\hline White & 30,520 & $65 \%$ & \multicolumn{2}{|c|}{ Research Doctorate } & 38 & Enrollment Profile: & High undergraduate \\
\hline Full-Time & 30,875 & $65 \%$ & \multirow{2}{*}{$\begin{array}{l}\text { Faculty } \\
\text { (Fall 2009) }\end{array}$} & \multirow{2}{*}{$\begin{array}{l}\text { Full- } \\
\text { Time }\end{array}$} & \multirow{2}{*}{ Part-Time } & Size and Setting: & Large four-year, primarily nonresidential \\
\hline Part-Time & 16,431 & $35 \%$ & & & & \multirow{2}{*}{ Basic: } & \multirow{2}{*}{$\begin{array}{c}\text { Research Universities } \\
\text { (very high research activity) }\end{array}$} \\
\hline Undergraduate & 35,834 & $76 \%$ & TOTAL & 1,618 & 320 & & \\
\hline Graduate & 9,273 & $20 \%$ & Tenure/T. Track & 1,115 & 79 & \multirow{2}{*}{ Elective Classification: } & Community Engagement: \\
\hline Unclassified & 2,199 & $5 \%$ & Other Faculty/Instr. & 503 & 241 & & Outreach \& Partnerships \\
\hline
\end{tabular}

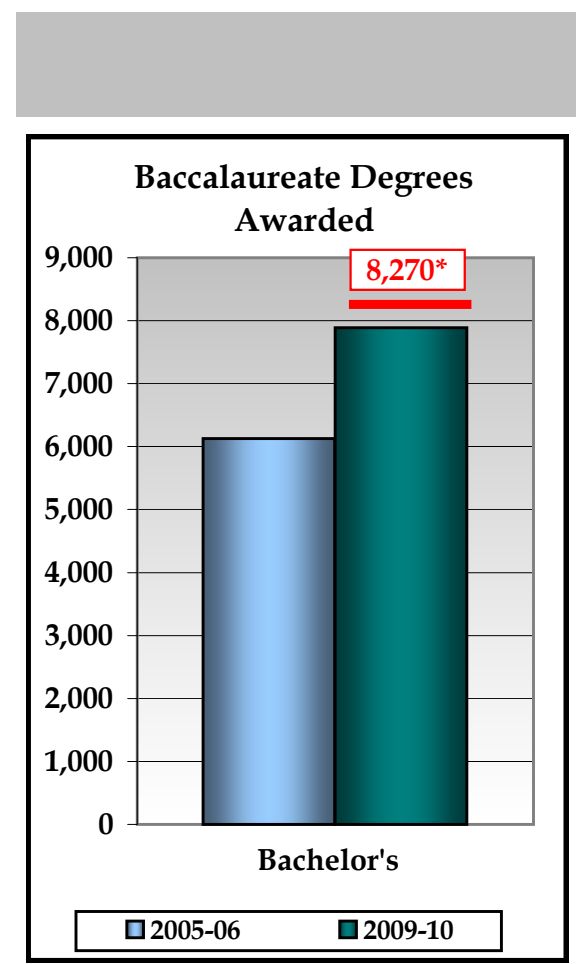

*2012-13 Targets for Degrees Awarded.

Note: All targets are based on 2010 University Workplans.
BOARD OF GOVERNORS - STATE UNIVERSITY SYSTEM GOAL 1: ACCESS TO AND PRODUCTION OF DEGREES

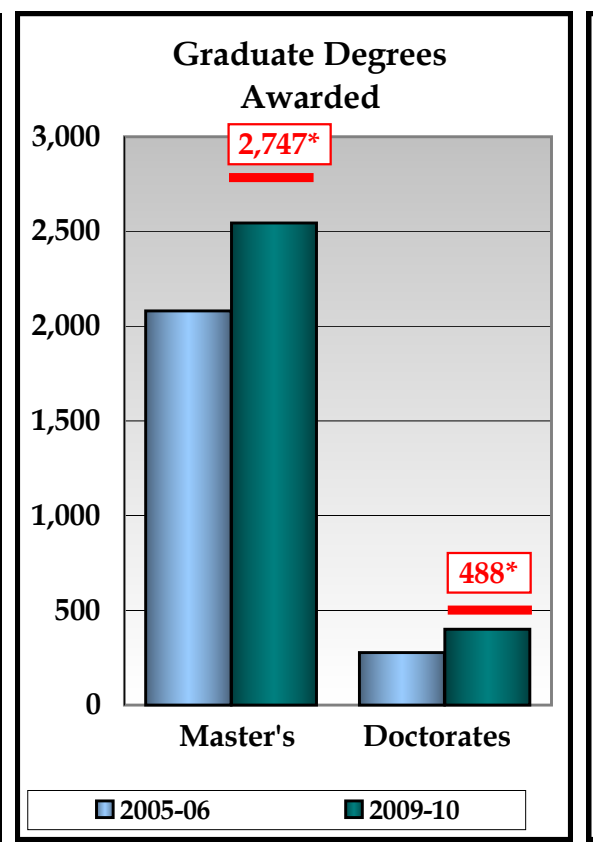

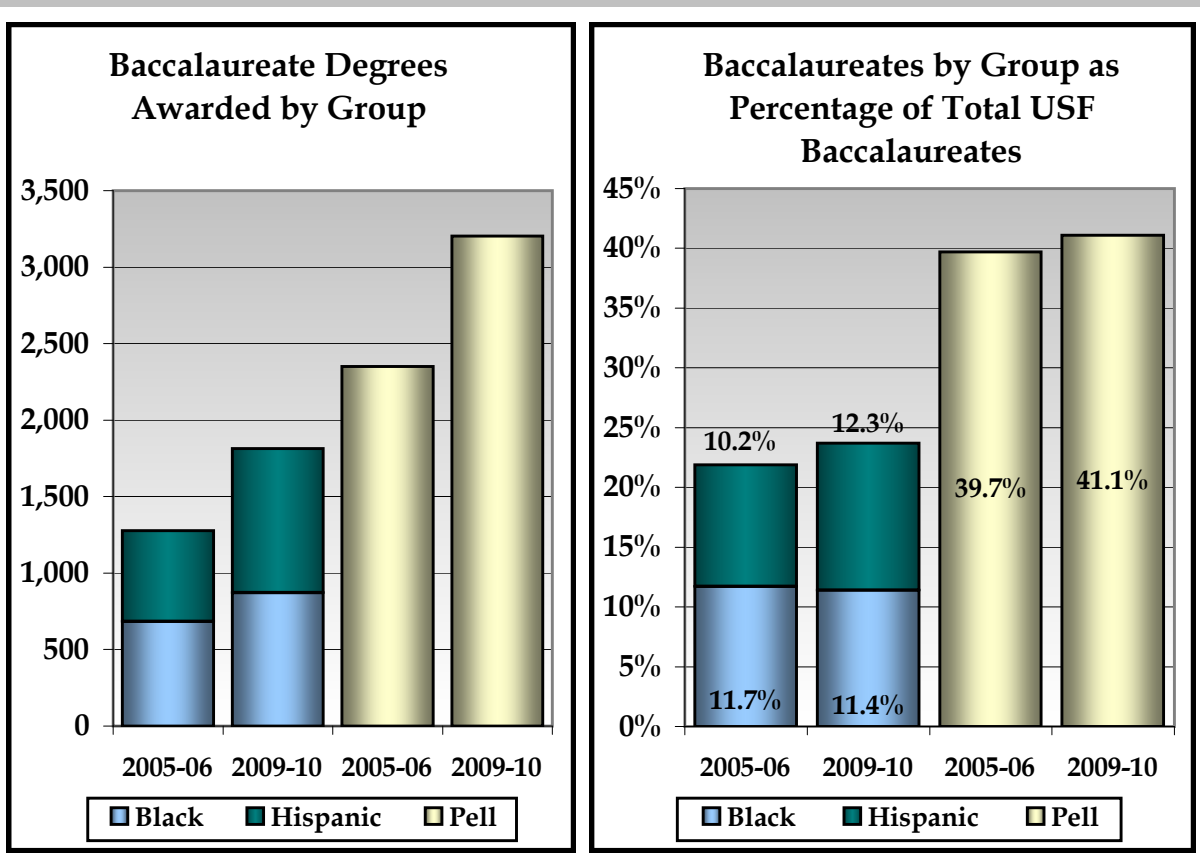

[2012-13 Targets for Baccalaureates By Group Reported in Volume II - Table 4I.]. 
BOARD OF GOVERNORS - STATE UNIVERSITY SYSTEM GOAL 2:

MEETING STATEWIDE PROFESSIONAL AND WORKFORCE NEEDS

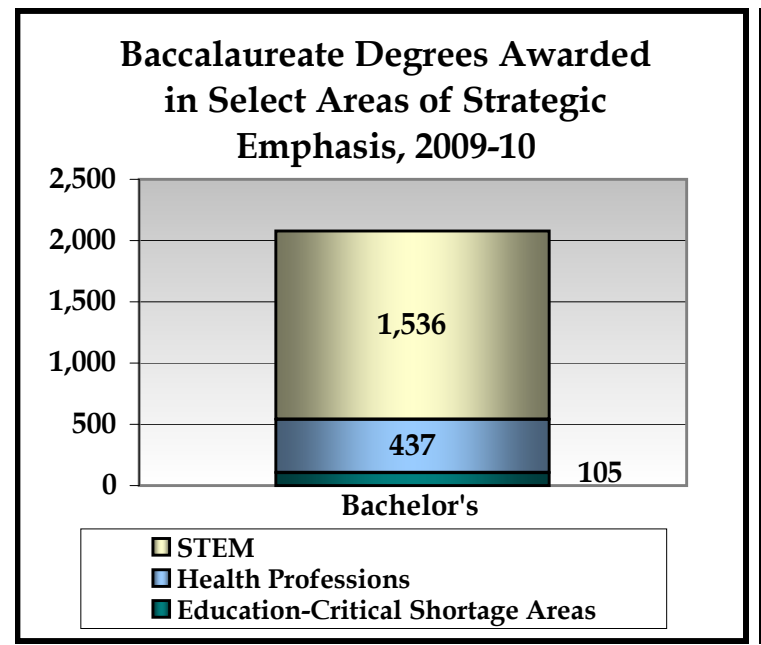

2012-13 Target: Increase (2008-09 Baseline: 1,942 Total)
Graduate Degrees Awarded in Select Areas of Strategic Emphasis, 2009-10

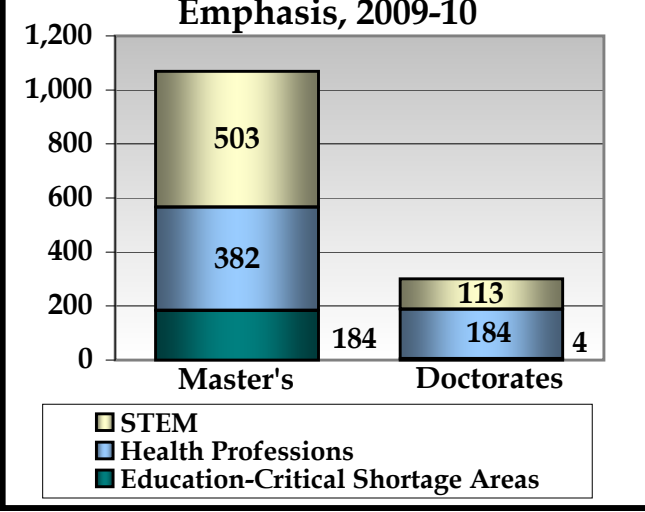

2012-13 Target: Increase (2008-09 Baseline: 1,258 Total)

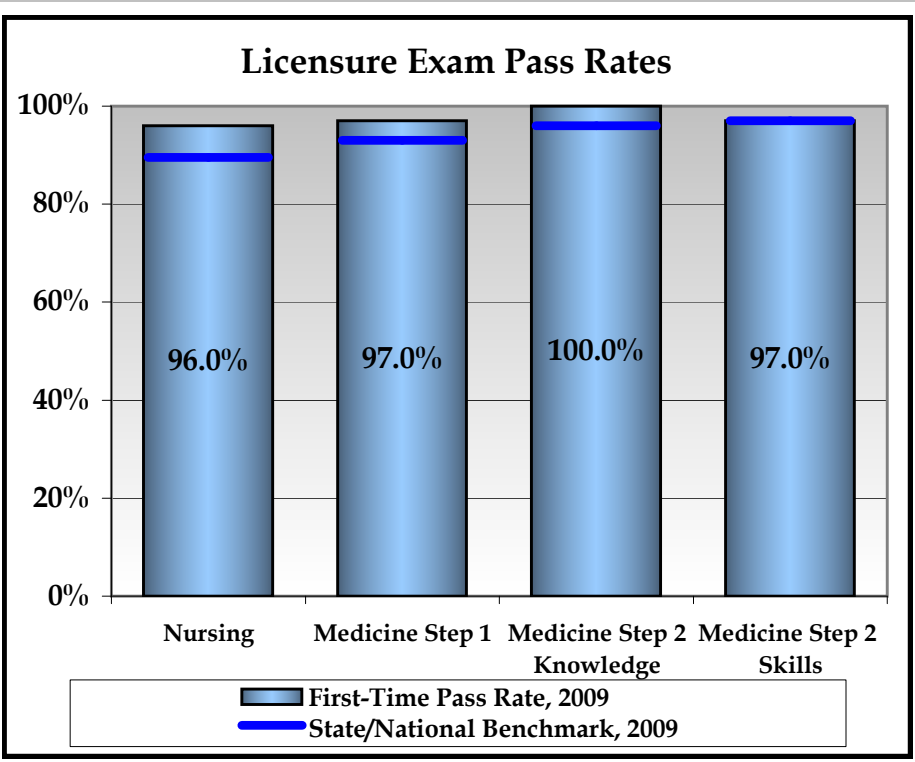

State/National Benchmark, 2009

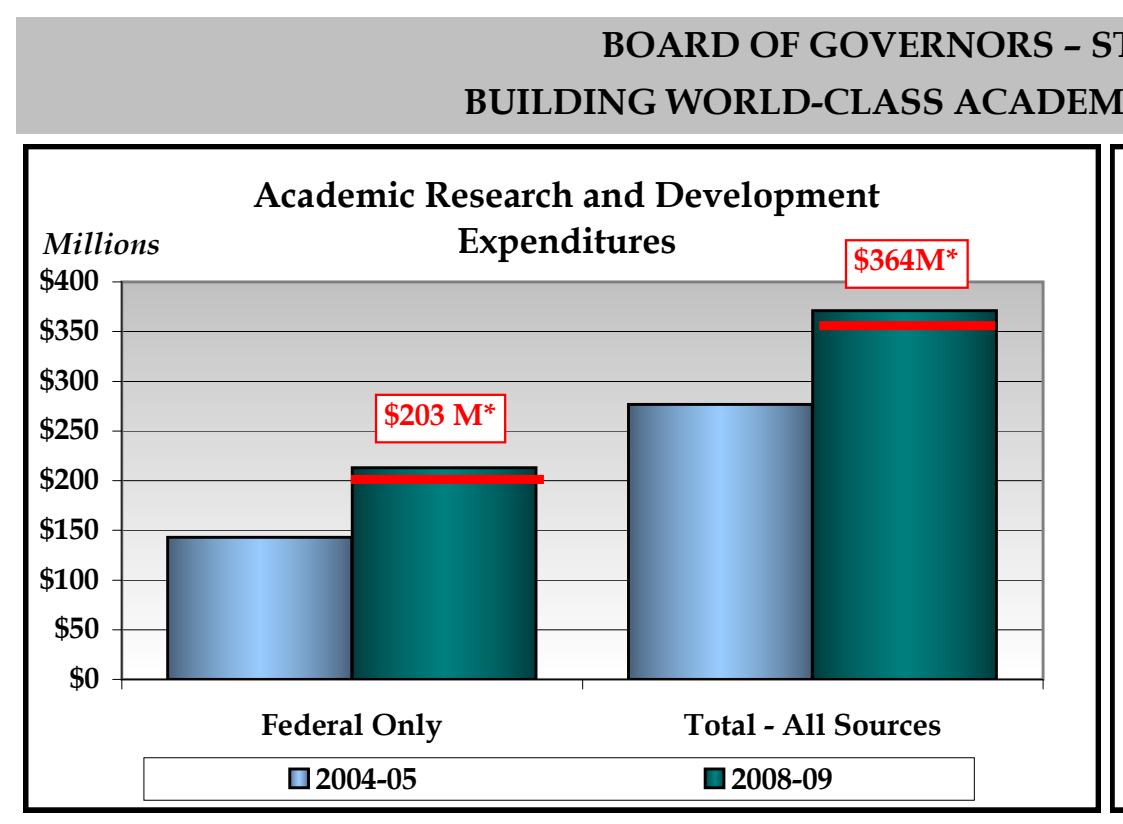

*2011-12 Targets for Research \& Development Expenditures.

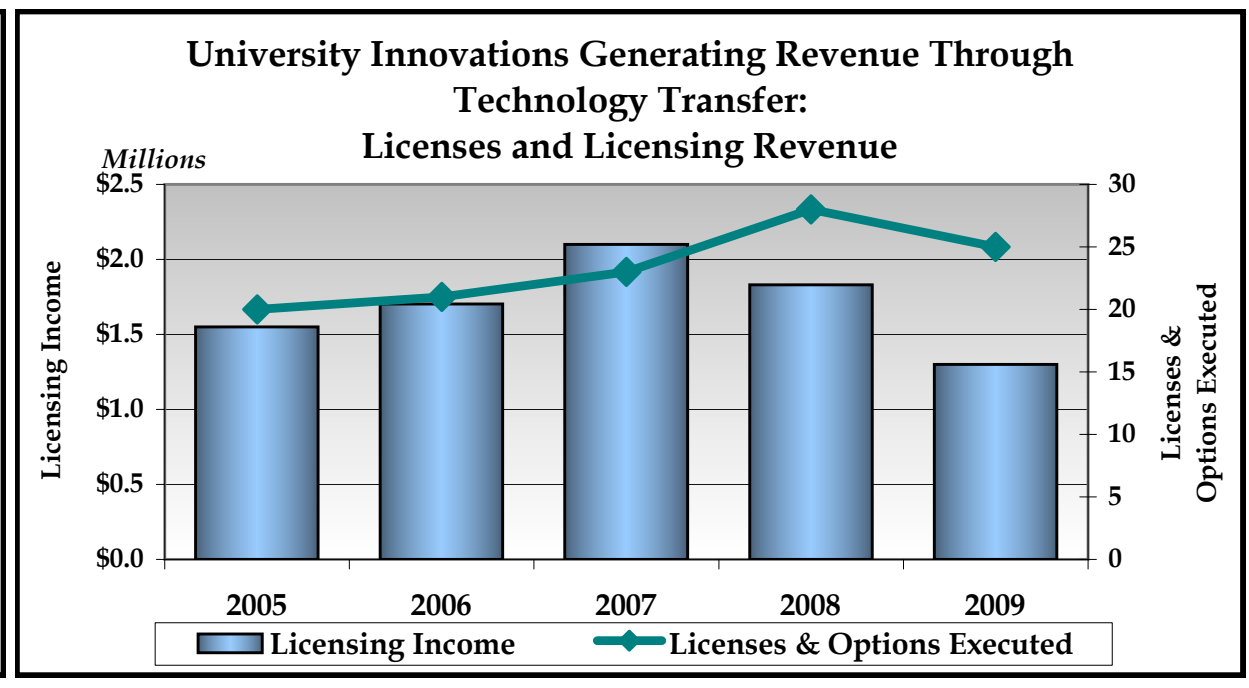

2011-12 Targets: Licenses - Increase (2008 Baseline = 23) Licensing Revenue - Increase (2008 Baseline $=\$ 2,099,712)$ 


\section{Select Data Tables from the 2009-2010 Annual Report}

* Peer choices should be noted. In cases in which peer data are not available for a specific metric, but are available for a related metric, an institution might want to note such in the "Comparison with Peers" row.

\begin{tabular}{|c|c|c|c|c|c|c|}
\hline Degrees Awarded & 2005-06 & 2006-07 & \multicolumn{2}{|l|}{ 2007-08 } & 2008-09 & 2009-10 \\
\hline Baccalaureate & 6,129 & 6,736 & \multicolumn{2}{|l|}{7,086} & 7,479 & 7,891 \\
\hline Master's and Specialist & 2,081 & 2,113 & \multicolumn{2}{|l|}{2,314} & 2,482 & 2,544 \\
\hline Research Doctoral & 184 & 223 & \multicolumn{2}{|l|}{229} & 248 & 244 \\
\hline Professional Doctoral & 93 & 122 & \multicolumn{2}{|l|}{143} & 154 & 156 \\
\hline \multirow{8}{*}{ Comparison with Peers* } & \multicolumn{6}{|c|}{$\begin{array}{l}\text { Peer Institutions: The Ohio State University, Rutgers University, University of Pittsburgh and Arizona State } \\
\text { University serve as peers to the USF System. The Ohio State University, Rutgers University and the University of } \\
\text { Pittsburgh are members of the Association of American Universities (Arizona State University is not an AAU } \\
\text { institution). The data used for comparison of metrics include each system, except when these data are not readily } \\
\text { available. In these cases the main campus is used for comparisons. Only the University of Pittsburgh and Rutgers } \\
\text { include regional campuses with significantly more than 3,000 students. } \\
\text { See http://www.ods.usf.edu/Plans/Strategic/docs/2010-10-07-Performance-Update-AAU.pdf for comparisons with } \\
\text { AAU public institutions, Florida research universities, and AAU prospects. } \\
\text { The USF System has gradually increased the number of degrees awarded in all categories over the last five years. In } \\
\text { comparison with its peers, the data in the table below show that in 2009-10, the USF System awarded more } \\
\text { baccalaureate, master's and specialist degrees than Rutgers and Pittsburgh, but fewer than Ohio State and Arizona } \\
\text { State. In other categories, the USF System awarded fewer degrees than its peers. However, all have larger enrollments } \\
\text { than the USF System except the University of Pittsburgh. }\end{array}$} \\
\hline & $\begin{array}{l}\text { Degrees Awarded } \\
(2009-10)\end{array}$ & USF System & $\begin{array}{c}\text { Arizona State } \\
\text { University }\end{array}$ & $\begin{array}{c}\text { Rutgers } \\
\text { University }\end{array}$ & Ohio State & $\begin{array}{c}\text { Univ. of } \\
\text { Pittsburgh }\end{array}$ \\
\hline & $\begin{array}{l}\text { System Enrollment } \\
\text { Headcount }\end{array}$ & 47,024 & 68,064 & 54,648 & 62,512 & 35,395 \\
\hline & System Enrollment FTE & 37,115 & 59,850 & 47,929 & 56,758 & 32,405 \\
\hline & BA/BS & $7,891(73 \%)$ & $11,810(72 \%)$ & $8,170(66 \%)$ & $9,880(70 \%)$ & $4,957(61 \%)$ \\
\hline & MA/MS/Sp. & $2,544(23 \%)$ & $3,914(24 \%)$ & $2,877(23 \%)$ & $2,695(19 \%)$ & $2,240(28 \%)$ \\
\hline & Res. Doc. & $244(2 \%)$ & $490(3 \%)$ & $484(5 \%)$ & 757 (5\%) & $400(5 \%)$ \\
\hline & Prof. Doc. & $156(1 \%)$ & $166(1 \%)$ & $783(6 \%)$ & $839(6 \%)$ & $544(6 \%)$ \\
\hline
\end{tabular}




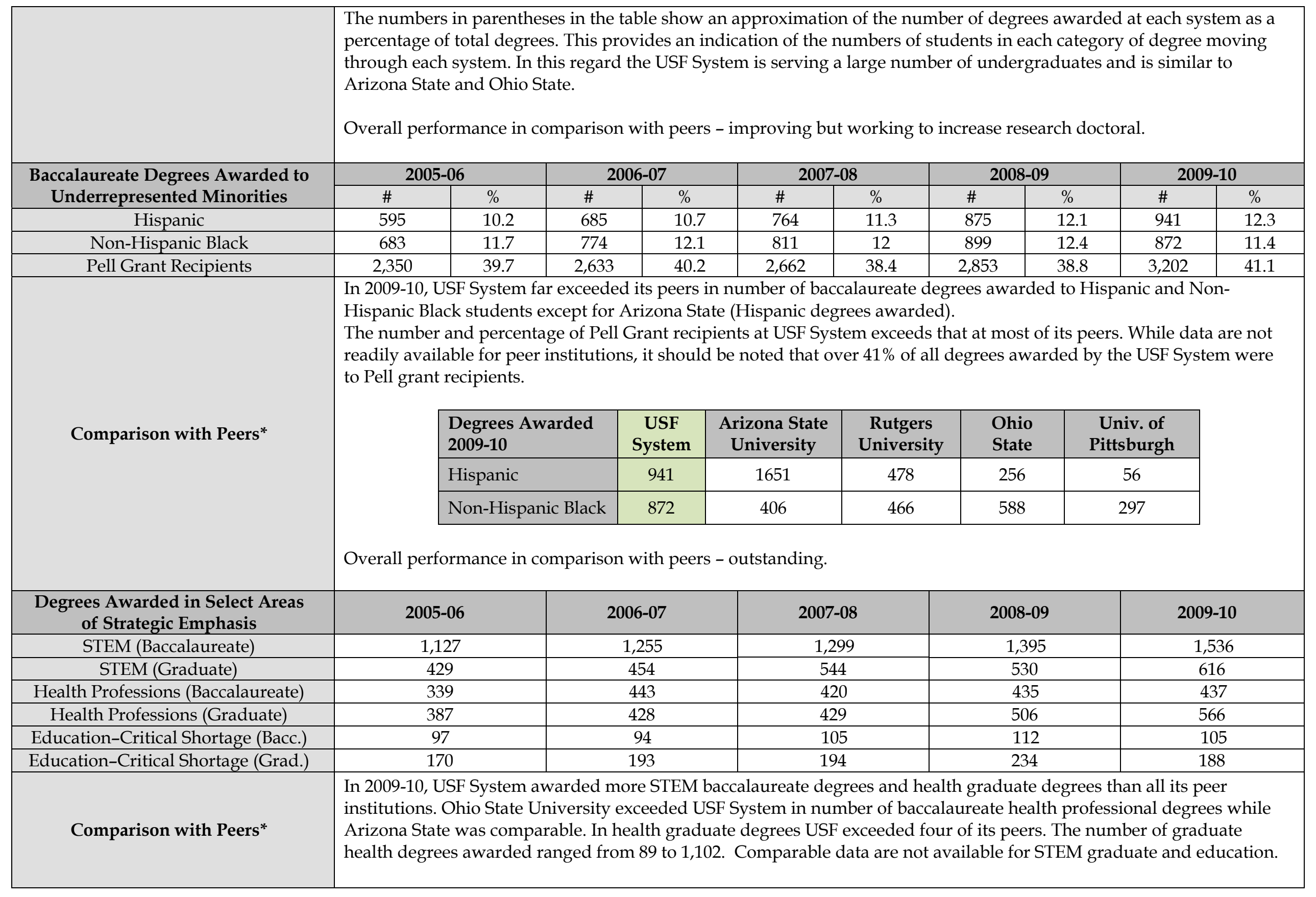




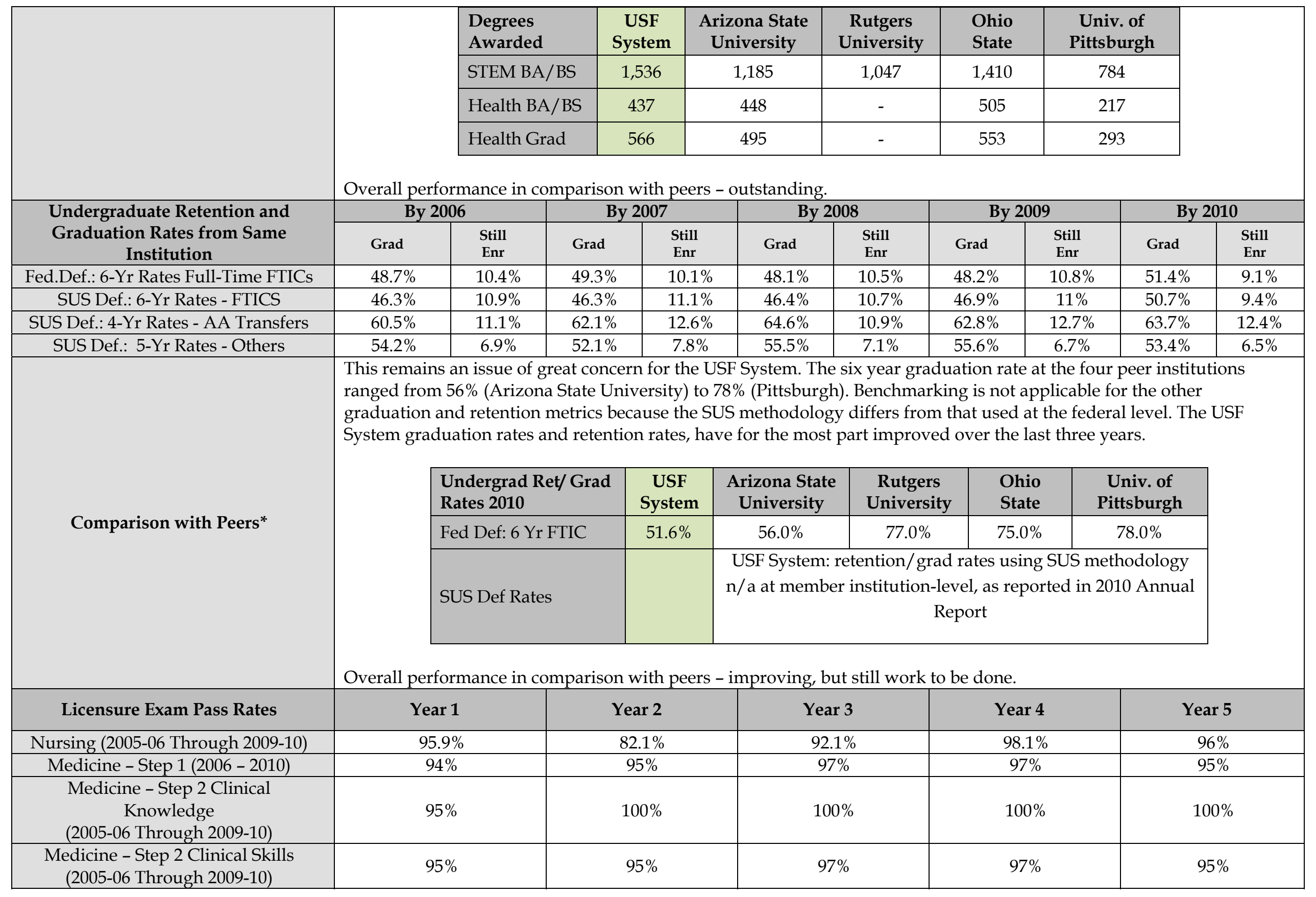




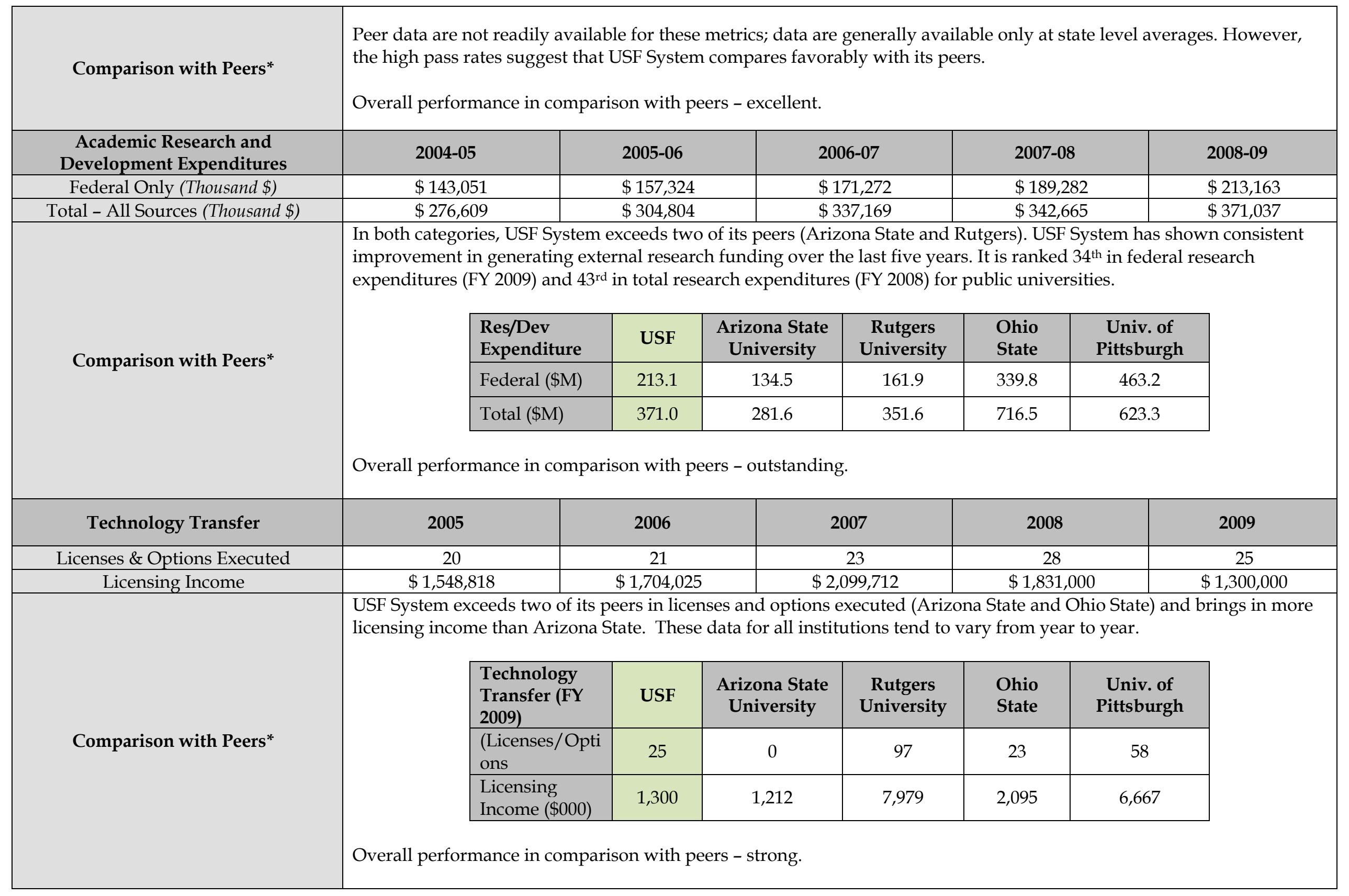




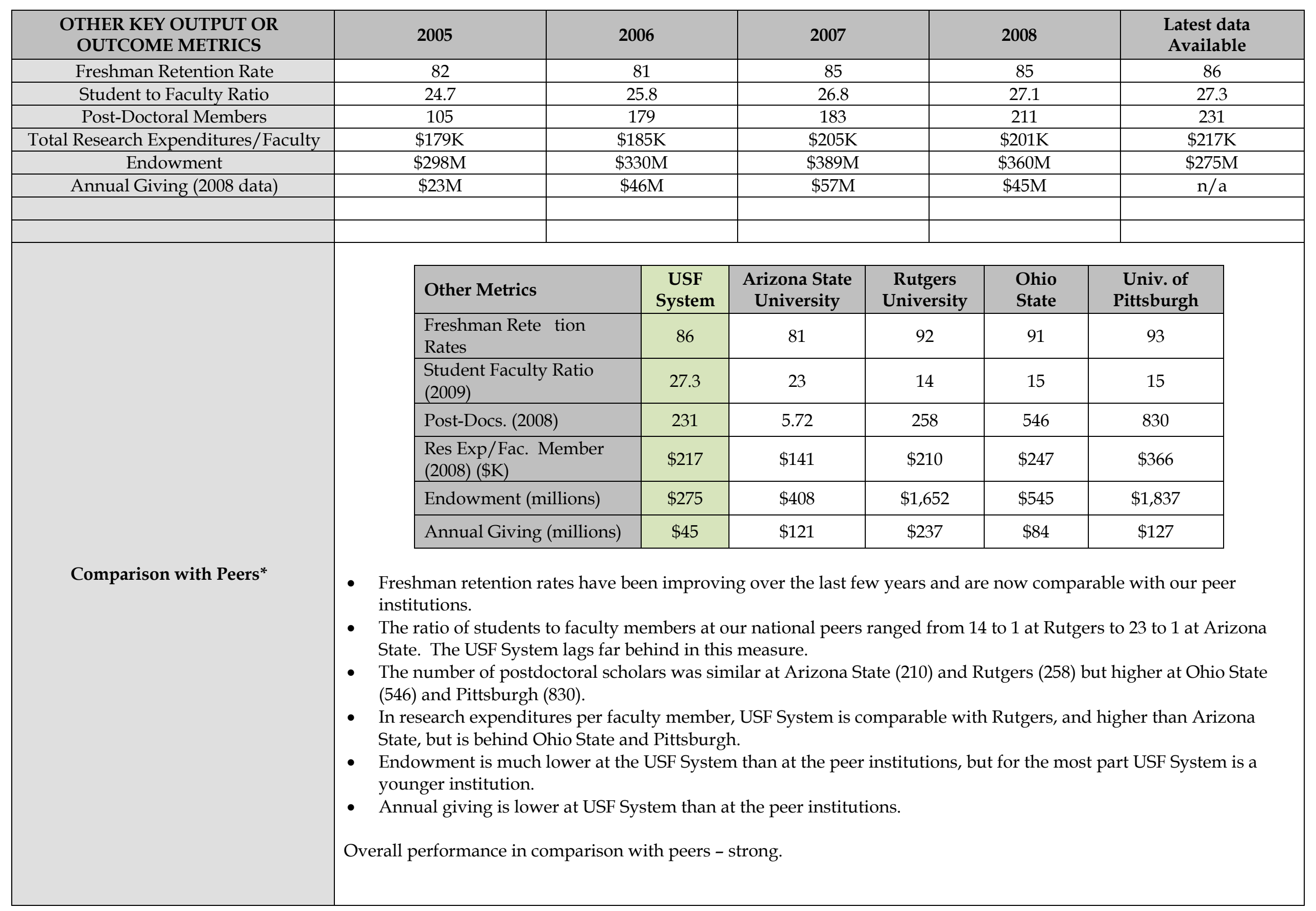


(1) Graduation Rates: While the six-year graduation rate for FTIC students has steadily improved since 2008 at USF System, from $48.1 \%$ to $51.6 \%$, it still remains relatively low in comparison with three of our peers. However, the strategic initiative highlighting student success and the investment of tuition dollars is now paying benefits with higher retention rates and more students graduating in a timely manner. This initiative continues to be a very high priority for the USF System.

(2) Infrastructure: An important challenge to USF System is its maintaining and developing its overall infrastructure. This entails not only its physical plant but also its academic support structures. There is, for example, a need for buildings and increased space at the three regional institutions as they develop their undergraduate programs. There is a shortage of residence halls, laboratories, and classrooms that limit access to the university. Similarly, academic infrastructure is under pressure. The libraries, as they move towards being a member of the Association of Research Libraries, need special attention, as too does the enhancement of the technological resources that lead to greater innovation.

(3) USF System Changes: As the USF System evolves and the four member institutions are separately accredited, issues of shared services and independent responsibilities come to the fore. These issues are being actively addressed to ensure that the USF System attains all the advantages of being a system while fostering the independent missions of each institution. At the USF System, this means focusing on student success at all academic levels while vigorously promoting research commensurate with our AAU goals at USF and differential missions at USF St. Petersburg, USF Sarasota-Manatee and USF Polytechnic as detailed in the four attached annual work plans. 


\section{UPDATES TO 2010 UNIVERSITY WORK PLAN}

[Please identify briefly any critical changes only to information provided in the 2010 University Work Plan that was not updated in the 2009-2010 Annual Report regarding the institution's strategic plan; institutional mission, vision, and strategic directions for the next five to ten years; current or aspirational peer institutions; windows of opportunity; or unique challenges.]

\section{Goal 1: Academic Excellence, Student Access, and Student Success}

The USF System remains committed to academic excellence, student access and student success. The USF System continues to develop a rich array of academic programs that are recognized regionally and nationally for their rigor, intellectual challenge, and high expectations. The USF System has increased access to these programs to qualified and diverse students and promotes student learning and success by supporting activities that are improving retention and graduation rates, employment and admission to graduate or professional schools. The USF System is seeking to lower average student debt loads, and raise student satisfaction in comparison with peer institutions.

\section{Goal 2: Impactful Research, Economic Leadership and Community Engagement}

The USF System continues to coordinate and promote: (i) research and innovation by providing information and services creating synergies among faculty across the System and fostering external partnerships; (ii) synergies among its institutions to become a major economic engine for the region and the state; (iii) increased production of graduates in high demand fields; (iv) increased applied research supported by the private sector; and (v) incubation of new companies, agencies, and occupations.

The USF System continues to challenge its institutions to increase community engagement by sharing best practices; USF and USF St. Petersburg have been designated Carnegie "Community Engaged" institutions.

\section{Goal 3: Increased Academic and Administrative Collaborations}

The USF System continues to provide leadership to increase academic and administrative collaborations among its institutions by establishing opportunities for students to enter graduate and professional programs across the USF System; by creating joint degree programs and innovative cross-institution curricula; by promoting joint research activities and other faculty development opportunities; by improving the efficiency, effectiveness, and functionality of System-wide administrative processes, systems, and technologies; and by the continuous improvement of those services, including increased user satisfaction.

\section{Goal 4: Open Communication and Effective Branding}

The USF System continues to develop a shared understanding of the USF System's common identity and brand promise and its relationship to the unique missions and distinctive identities of its member institutions for both internal and external audiences.

\section{Goal 5: Expanded and Diversified Resources}

The USF System continues to expand and diversify its resource base to maintain financial sustainability of its member institutions and to assist them in meeting their distinctive missions. The USF System continues to focus on its endowment, its annual giving levels, its research grants and contracts, its revenues from auxiliaries and Direct Service Organizations, and its overall efficiency. In partnership with the leadership of its institutions, it continues to work with Florida's legislature to increase investment in higher education and to expand the fiscal flexibility of the USF System and its member institutions.

The USF System: The USF System coordinates activities of its four member institutions and as a unified entity provides:

- Enhanced access for students

- Distinctiveness while optimizing potential

- Greater choice to meet student and academic needs 
- Broader advocacy

- Efficiencies, both academic and economic

- Commitment to meeting local needs

- Leveraging our combined strength through collaboration

- A unified brand yielding identity and impact.

The USF System works to enhance and facilitate the individual missions of all four member institutions. Two member institutions currently have separate IPEDS reporting, USF (in Tampa) and USF St. Petersburg, and are accredited by SACS. USF Sarasota-Manatee should be SACS accredited in June 2011 and USF Polytechnic in 2012. Under the Carnegie Foundation for the Advancement of Teaching classification: USF is a doctoral university with very high research activity; USF ST. Petersburg, USF Sarasota-Manatee and USF Polytechnic are each classified as masters, medium level. USF and USF St. Petersburg are also Carnegie Community Engaged institutions.

With the development of the USF System new peers have been established for USF St. Petersburg, USF Sarasota-Manatee and USF Polytechnic (see individual work plans). In addition, new peers have been identified to reflect components of USF System. Within this framework, USF remains firmly committed to its research goals, AAU aspirations and regularly compares itself with major research institutions (http:// www.ods.usf.edu/Plans/Strategic/docs/2010-10-07-Performance-Update-AAU.pdf). 
CAVP Academic Coordination Project (List degree programs recommended for new collaborative or joint delivery model or other corrective action, as well as any degree programs recommended for continuation but for which university and Board staff have not reached agreement on the sufficiency of the rationale.)

\begin{tabular}{|c|c|c|c|c|}
\hline $\begin{array}{l}\text { Program } \\
\text { Level }\end{array}$ & $\begin{array}{l}\text { 6-Digit } \\
\text { CIP } \\
\text { Code }\end{array}$ & Program Title & $\begin{array}{c}\text { Category (i.e., } \\
\text { Collaborative Model, } \\
\text { Corrective Action, or } \\
\text { Proposed } \\
\text { Continuation) }\end{array}$ & Proposed Action \\
\hline $\mathrm{D}$ & 14.0501 & Biomedical Engineering & $\begin{array}{l}\text { This program is in a } \\
\text { unit that is highly } \\
\text { productive; Chemical } \\
\text { Engineering. The } \\
\text { department awards } \\
\text { degrees for BS, MS and } \\
\text { Ph.D. in Chemical } \\
\text { Engineering. }\end{array}$ & $\begin{array}{l}\text { This program started in } 2005 \text { with } \\
\text { no new resources and has produced } \\
1.6 \text { degrees per year, on average. } \\
\text { Since } 2009 \text { the college has hired four } \\
\text { new faculty in this program. With } \\
\text { increased involvement of the } \\
\text { Medical School we expect the } \\
\text { number of graduates to increase } \\
\text { beyond the threshhold. }\end{array}$ \\
\hline $\mathrm{D}$ & 14.1901 & Mechanical Engineering & $\begin{array}{l}\text { The program is in a unit } \\
\text { that awards a high } \\
\text { number of degrees at } \\
\text { the BS and MS level. } \\
\text { The program also } \\
\text { delivers a high number } \\
\text { of student credit hours } \\
\text { and currently it has a } \\
\text { high number of } \\
\text { enrolled Ph.D. students. }\end{array}$ & $\begin{array}{l}\text { There is a corrective action plan in } \\
\text { place to improve graduation rates. } \\
\text { Twenty-one new Ph.D. students } \\
\text { were admitted to the department } \\
\text { during AY 2010-2011. Current } \\
\text { Ph.D. enrollment is close to 40; } \\
\text { going forward, this will result in } \\
\text { significant increase in Ph.D. degrees } \\
\text { awarded. }\end{array}$ \\
\hline $\mathrm{D}$ & 45.0401 & Criminology & $\begin{array}{l}\text { The program is in a unit } \\
\text { that serves a large } \\
\text { number of } \\
\text { undergraduate students } \\
\text { (1200) and graduate } \\
\text { students (99) with only } \\
12 \text { faculty. Faculty } \\
\text { scholarly productivity is } \\
\text { ranked in top ten for the } \\
\text { discipline. }\end{array}$ & $\begin{array}{l}\text { Curricular revisions are underway } \\
\text { to: implement a 3-member faculty } \\
\text { committee to promote timely degree } \\
\text { completion; reviewing departmenta } \\
\text { policies regarding timeline for } \\
\text { degree completion. A plan is in } \\
\text { place to increase faculty hiring. In } \\
\text { addition, a three-year plan will be } \\
\text { developed to increase graduate } \\
\text { stipends }\end{array}$ \\
\hline $\mathrm{D}$ & 50.0901 & Music, General & $\begin{array}{l}\text { This is the Ph.D. } \\
\text { program in Music } \\
\text { Education. }\end{array}$ & $\begin{array}{l}\text { The program has increased the } \\
\text { number of enrolled students and } \\
\text { additional recruitment positions the } \\
\text { program to meet and exceed the } \\
\text { minimum requirement for average } \\
\text { graduation rate. }\end{array}$ \\
\hline EdS & 13.0401 & $\begin{array}{l}\text { Education } \\
\text { Administration/Ldrshp, Gnrl }\end{array}$ & $\begin{array}{l}\text { There is no additional } \\
\text { pecuniary or non- } \\
\text { pecuniary cost to the } \\
\text { department as these } \\
\text { students enroll in the } \\
\text { same courses as those } \\
\text { pursuing a doctorate; in } \\
\text { cases where doctoral } \\
\text { students fail to make } \\
\text { satisfactory progress in }\end{array}$ & $\begin{array}{l}\text { We are in the process of submitting } \\
\text { a new folio to the Florida } \\
\text { Department of Education that will } \\
\text { add principal certification track to } \\
\text { our Educational Specialist degree. } \\
\text { This will replace our current } \\
\text { principal certificate modified } \\
\text { program which is a non-degree } \\
\text { program. We anticipate an increase } \\
\text { in enrollment with this change. }\end{array}$ \\
\hline
\end{tabular}




\begin{tabular}{|c|c|c|c|c|}
\hline & & & $\begin{array}{l}\text { their program, the } \\
\text { specialist degree } \\
\text { provides an alternative } \\
\text { to non-degree } \\
\text { completion; the } \\
\text { educational specialists } \\
\text { degree prepares } \\
\text { students to teach at the } \\
\text { community college } \\
\text { level without attaining } \\
\text { the doctorate; and, the } \\
\text { educational specialist } \\
\text { degree provides an } \\
\text { avenue for students } \\
\text { who already have a } \\
\text { master's degree, but do } \\
\text { not want to pursue a } \\
\text { doctorate. }\end{array}$ & \\
\hline M & 05.0102 & American Studies & $\begin{array}{l}\text { The department is in } \\
\text { the midst of executing a } \\
\text { plan to grow this } \\
\text { program beyond critical } \\
\text { levels as part of their } \\
\text { efforts to "re-invent" } \\
\text { American Studies. This } \\
\text { also provides excellent } \\
\text { support for the } \\
\text { university's newly } \\
\text { articulated global } \\
\text { strategy. }\end{array}$ & $\begin{array}{l}\text { We expect enrollment to exceed } \\
\text { required levels within two years. } \\
\text { This program also provides } \\
\text { significant support for the } \\
\text { university's general education } \\
\text { program in the areas of Humanities } \\
\text { and Fine Arts. }\end{array}$ \\
\hline M & 05.0207 & Women Studies & $\begin{array}{l}\text { The Department of } \\
\text { Women's Studies at } \\
\text { USF has undergone a } \\
\text { radical transformation } \\
\text { in the past year with the } \\
\text { renaming of the } \\
\text { department (now the } \\
\text { more inclusive } \\
\text { Women's and Gender } \\
\text { Studies) and all new } \\
\text { faculty. }\end{array}$ & $\begin{array}{l}\text { In years past it had a large and } \\
\text { successful Master's Program and } \\
\text { within a year or two it will have one } \\
\text { once again. The department is } \\
\text { currently aggressively recruiting } \\
\text { affiliate faculty and new students. }\end{array}$ \\
\hline
\end{tabular}


New Academic Degree Program Proposals - Next Three Years (Program development goals need to align with the institutional strategic plan and System priorities.)

\begin{tabular}{|c|c|c|c|c|}
\hline $\begin{array}{l}\text { Proposed Date of } \\
\text { Submission to } \\
\text { University Board of } \\
\text { Trustees } \\
\end{array}$ & $\begin{array}{c}\text { Program } \\
\text { Level }\end{array}$ & $\begin{array}{l}\text { 6-Digit CIP } \\
\text { Code }\end{array}$ & Program Title & $\begin{array}{c}\text { Comments } \\
\text { (Including Proposed } \\
\text { Implementation Date) }\end{array}$ \\
\hline \multicolumn{5}{|l|}{ USF } \\
\hline Dec 2011 & B & 51.000 & Health Science & 2012 Health \\
\hline Dec 2011 & B & 51.1599 & Behavioral Healthcare & 2012 Health \\
\hline Dec 2011 & M & 09.0903 & Advertising & 2012 \\
\hline Dec 2011 & M & 30.2001 & $\begin{array}{c}\text { Diplomacy and Strategic } \\
\text { Studies }\end{array}$ & 2012 Global \\
\hline Spring 2012 & M & 31.0504 & Sport Management & 2012 \\
\hline Dec 2011 & M & 51.2707 & Health Informatics & 2012 Health \\
\hline Dec 2011 & M & 26.0907 & $\begin{array}{c}\text { Diabetes and AutoImmune } \\
\text { Diseases }\end{array}$ & 2012 Health \\
\hline Dec 2011 & RD & 14.1407 & Environmental Engineering & 2012 STEM \\
\hline Dec 2011 & RD & 42.2814 & Applied Behavioral Analysis & 2012 Health \\
\hline Dec 2011 & RD & 51.2314 & Rehabilitation Sciences & 2013 Health \\
\hline \multicolumn{5}{|l|}{$\begin{array}{l}\text { USF St. } \\
\text { Petersburg }\end{array}$} \\
\hline Dec 2011 & M & 13.1203 & $\begin{array}{l}\text { Middle Grades STEM } \\
\text { Education }\end{array}$ & 2012 Education \\
\hline Spring 2012 & M & 42.0101 & Psychology & 2012 Health \\
\hline \multicolumn{5}{|l|}{$\begin{array}{l}\text { USF Sarasota- } \\
\text { Manatee }\end{array}$} \\
\hline Dec 2011 & M & 13.1305 & Secondary English Education & 2012 Education \\
\hline Spring 2012 & M & 13.1201 & Adult Education & 2012 Education \\
\hline \multicolumn{5}{|l|}{ USF Polytechnic } \\
\hline Spring 2012 & B & 14.2701 & Systems Engineering & $\begin{array}{l}\text { Upon SACs Approval } \\
\text { STEM }\end{array}$ \\
\hline Spring 2012 & B & 52.0304 & $\begin{array}{l}\text { Accounting and Financial } \\
\text { Management }\end{array}$ & $\begin{array}{l}\text { Upon SACs Approval } \\
\text { STEM }\end{array}$ \\
\hline Spring 2012 & B & 51.2706 & $\begin{array}{l}\text { Health Information } \\
\text { Management }\end{array}$ & $\begin{array}{l}\text { Upon SACs Approval } \\
\text { Health }\end{array}$ \\
\hline
\end{tabular}




\section{Enrollment Planning}

Please explain briefly any planned changes in enrollment patterns in the next five years, with rationale (e.g., more emphasis on enrolling FCS AA transfers; enrollment of more out-of-state students; enrollment of more FTICs as the institution builds out a more residential experience for undergraduates; maintain undergraduate enrollment with more growth at graduate level to align with institutional mission; plan to maintain current enrollment with more emphasis on improving graduation rates; etc.).

The USF System is committed to academic excellence, student access and student success. The USF System continues to develop a rich array of academic programs that are recognized regionally and nationally for their rigor, intellectual challenge, and high expectations. The USF System has increased access to these programs to qualified and diverse students and promotes student learning and success by supporting activities that are improving retention and graduation rates, employment and admission to graduate or professional schools. The USF System is seeking to lower average student debt loads, and raise student satisfaction in comparison with peer institutions.

USF's undergraduate enrollment will be held relatively stable as undergraduate education is aligned more with USF St. Petersburg, USF Sarasota-Manatee and USF Polytechnic reflecting the distinctive missions of each institution. USF St. Petersburg is the only regional institution that currently admits freshmen, although USF Sarasota-Manatee and USF Polytechnic are pursuing four-year programs to include freshmen and women, and sophomores in the next two years. This will increase undergraduate teaching and learning opportunities across the system, through expanded integrated, interdisciplinary initiatives and global activities. Data on undergraduate enrollment and retention are regularly collected (e.g., specific cohort retention and graduation rates, admit yield rates, enrollment rates).

1. Annual FTE enrollment plans by level, site, and residency for tuition purposes in the format provided in the template on the next pages.

2. These are only to include fundable FTE enrollments. So, for example, out-of-state profile admits should not be included in the out-of-state data.

3. Remember that Pharm.D., Law, and other Professional Doctorates (per the recently changed IPEDS definitions) should be counted as Grad II enrollments.

4. An explanation of over-enrollment is required for any level in which the 2010-11 funded enrollment plan lagged actual 2010-11 enrollment by more than 5\% (Section 1011.90, F.S.).

With the absence of increased state appropriations for growth, the funded plan has not grown at the same pace as actual enrollment. Additional reasons for the enrollment growth are various, and include the following:

- USF's enrollment response reflects significant progress in addressing SUS priorities, including: providing increased access and production of degrees which is reflected in increases at all levels, increasing world-class research efforts (which partially explains the large increase in Grad II numbers), and meeting targeted program and critical statewide work force needs, such as health care (including nursing and pharmacy), engineering and technology, and education (all of which have increased enrollment at USF).

- A permanent revenue neutral shift would reduce magnitude of variance. And obviously, the lack of distribution of funded FTE by the Legislature for three years is a major factor. 


\begin{tabular}{|c|c|c|c|c|c|c|c|c|}
\hline \multicolumn{9}{|c|}{$\begin{array}{l}\text { Enrollment Plan Proposal - All State-Fundable FTE Enrollments } \\
\text { (Except Medical/Dental/Veterinary Enrollments) }\end{array}$} \\
\hline $\begin{array}{l}\text { For entire } \\
\text { institution }\end{array}$ & Funded & Estimated & Funded & Estimated & Estimated & Estimated & Estimated & \multirow{2}{*}{$\begin{array}{c}\text { 5-Year } \\
\text { Projected } \\
\text { Average } \\
\text { Annual } \\
\text { Growth } \\
\text { Rate }\end{array}$} \\
\hline FTE & 2010-11 & 2010-11 & 2011-12 & 2011-12 & 2012-13 & 2014-15 & 2016-17 & \\
\hline $\begin{array}{c}\text { FL } \\
\text { Resident } \\
\text { Lower }\end{array}$ & 9,275 & 8,997 & 9,275 & 8,962 & 9,175 & 9,433 & 9,792 & $1.8 \%$ \\
\hline $\begin{array}{c}\text { FL } \\
\text { Resident } \\
\text { Upper }\end{array}$ & 12,777 & 13,600 & 12,777 & 13,863 & 14,208 & 14,949 & 15,876 & $2.7 \%$ \\
\hline $\begin{array}{c}\text { FL } \\
\text { Resident } \\
\text { Grad I }\end{array}$ & 3,185 & 2,815 & 3,185 & 3,014 & 3,146 & 3,381 & 3,678 & $4.1 \%$ \\
\hline $\begin{array}{c}\text { FL } \\
\text { Resident } \\
\text { Grad II }\end{array}$ & 622 & 788 & 622 & 800 & 832 & 899 & 970 & $3.9 \%$ \\
\hline $\begin{array}{l}\text { Total FL } \\
\text { Resident }\end{array}$ & 25,859 & 26,200 & 25,859 & 26,639 & 27,361 & 28,661 & 30,316 & $2.6 \%$ \\
\hline $\begin{array}{l}\text { Non-Res. } \\
\text { Lower }\end{array}$ & & 395 & & 452 & 521 & 597 & 695 & $9.0 \%$ \\
\hline $\begin{array}{l}\text { Non-Res. } \\
\text { Upper }\end{array}$ & & 426 & & 431 & 449 & 470 & 512 & $3.5 \%$ \\
\hline $\begin{array}{c}\text { Non-Res. } \\
\text { Grad I }\end{array}$ & & 340 & & 352 & 370 & 390 & 419 & $3.5 \%$ \\
\hline $\begin{array}{c}\text { Non-Res. } \\
\text { Grad II }\end{array}$ & & 427 & & 430 & 440 & 450 & 460 & $1.4 \%$ \\
\hline $\begin{array}{l}\text { Total Non- } \\
\text { Res. }\end{array}$ & 1,302 & 1,588 & 1,302 & 1,470 & 1,665 & 1,903 & 2,085 & $7.2 \%$ \\
\hline $\begin{array}{l}\text { Total } \\
\text { Lower }\end{array}$ & & & & & & & & \\
\hline $\begin{array}{l}\text { Total } \\
\text { Upper }\end{array}$ & & 9,392 & & 9,414 & 9,696 & 10,030 & 10,487 & $2.2 \%$ \\
\hline $\begin{array}{c}\text { Total Grad } \\
\text { I }\end{array}$ & & 14,026 & & 14,294 & 14,657 & 15,419 & 16,388 & $2.8 \%$ \\
\hline $\begin{array}{c}\text { Total Grad } \\
\text { II }\end{array}$ & & 3,155 & & 3,366 & 3,516 & 3,771 & 4,096 & $4.0 \%$ \\
\hline Total FTE & & 1,215 & & 1,230 & 1,272 & 1,349 & 1,430 & $3.1 \%$ \\
\hline
\end{tabular}




\begin{tabular}{|c|c|c|c|c|c|c|c|c|}
\hline \multicolumn{9}{|c|}{ Enrollment Plan Proposal - Medical/Dental/Veterinary State-Fundable Enrollments } \\
\hline $\begin{array}{l}\text { For entire } \\
\text { institution }\end{array}$ & Funded & Estimated & Funded & Estimated & Estimated & Estimated & Estimated & \multirow{2}{*}{$\begin{array}{c}\text { 5-Year } \\
\text { Projected } \\
\text { Average } \\
\text { Annual } \\
\text { Growth } \\
\text { Rate }\end{array}$} \\
\hline Headcount & 2010-11 & 2010-11 & 2011-12 & 2011-12 & 2012-13 & 2014-15 & 2016-17 & \\
\hline $\begin{array}{c}\text { FL Resident } \\
\text { Medical } \\
\text { Headcount }\end{array}$ & 480.0 & 458.0 & 480.0 & 460.0 & 460.0 & 460.0 & 460.0 & $0.2 \%$ \\
\hline $\begin{array}{c}\text { Non-Res. } \\
\text { Medical } \\
\text { Headcount }\end{array}$ & & 17.0 & & 20.0 & 20.0 & 20.0 & 20.0 & $0.2 \%$ \\
\hline $\begin{array}{c}\text { Total Medical } \\
\text { Headcount }\end{array}$ & 480.0 & 475.0 & 480.0 & 480.0 & 480.0 & 480.0 & 480.0 & $0.2 \%$ \\
\hline $\begin{array}{c}\text { FL Resident } \\
\text { Dentistry } \\
\text { Headcount }\end{array}$ & & & & & & & & \\
\hline $\begin{array}{c}\text { Non-Res. } \\
\text { Dentistry } \\
\text { Headcount }\end{array}$ & & & & & & & & \\
\hline $\begin{array}{c}\text { Total } \\
\text { Dentistry } \\
\text { Headcount }\end{array}$ & & & & & & & & \\
\hline $\begin{array}{c}\text { FL Resident } \\
\text { Veterinary } \\
\text { Headcount }\end{array}$ & & & & & & & & \\
\hline $\begin{array}{l}\text { Non-Res. } \\
\text { Veterinary } \\
\text { Headcount } \\
\end{array}$ & & & & & & & & \\
\hline $\begin{array}{c}\text { Total } \\
\text { Veterinary } \\
\text { Headcount } \\
\end{array}$ & & & & & & & & \\
\hline $\begin{array}{c}\text { FL Resident } \\
\text { Pharmacy } \\
\text { Headcount }\end{array}$ & 0 & 0 & & 50.0 & 225.0 & 375.0 & 400.0 & $140.0 \%$ \\
\hline $\begin{array}{l}\text { Non-Res. } \\
\text { Pharmacy } \\
\text { Headcount }\end{array}$ & & 0 & & - & - & - & - & $0.0 \%$ \\
\hline $\begin{array}{c}\text { Total } \\
\text { Pharmacy } \\
\text { Headcount }\end{array}$ & 0 & 0 & & 50.0 & 225.0 & 375.0 & 400.0 & $140.0 \%$ \\
\hline
\end{tabular}

[This medical headcount is MD-only, not all HSC enrollments.] 


\section{SITE: USF Health Science Center}

\begin{tabular}{|c|c|c|c|c|c|c|c|c|}
\hline USF-HSC & Funded & Estimated & Funded & Estimated & Estimated & Estimated & Estimated & \\
\hline FTE & 2010-11 & 2010-11 & 2011-12 & 2011-12 & 2012-13 & 2014-15 & 2016-17 & $\begin{array}{c}\text { Projected } \\
\text { Average } \\
\text { Annual } \\
\text { Growth Rate }\end{array}$ \\
\hline $\begin{array}{c}\text { FL } \\
\text { Resident } \\
\text { Lower }\end{array}$ & 103 & 376 & 103 & 357 & 368 & 370 & 372 & $0.8 \%$ \\
\hline $\begin{array}{c}\text { FL } \\
\text { Resident } \\
\text { Upper }\end{array}$ & 584 & 928 & 584 & 940 & 946 & 962 & 977 & $0.8 \%$ \\
\hline $\begin{array}{c}\text { FL } \\
\text { Resident } \\
\text { Grad I }\end{array}$ & 495 & 847 & 495 & 916 & 889 & 891 & 893 & $-0.5 \%$ \\
\hline $\begin{array}{c}\text { FL } \\
\text { Resident } \\
\text { Grad II } \\
\end{array}$ & 232 & 247 & 232 & 246 & 258 & 266 & 267 & $1.6 \%$ \\
\hline $\begin{array}{l}\text { Total FL } \\
\text { Resident }\end{array}$ & 1,414 & 2,398 & 1,414 & 2,459 & 2,461 & 2,489 & 2,509 & $0.4 \%$ \\
\hline $\begin{array}{l}\text { Non-res } \\
\text { Lower }\end{array}$ & & 11 & & 11 & 12 & 12 & 12 & $0.3 \%$ \\
\hline $\begin{array}{l}\text { Non-res } \\
\text { Upper }\end{array}$ & & 24 & & 23 & 23 & 24 & 24 & $0.5 \%$ \\
\hline $\begin{array}{l}\text { Non-res } \\
\text { Grad I }\end{array}$ & & 127 & & 100 & 97 & 96 & 95 & $-1.1 \%$ \\
\hline $\begin{array}{l}\text { Non-res } \\
\text { Grad II }\end{array}$ & & 48 & & 27 & 28 & 27 & 27 & $0.3 \%$ \\
\hline $\begin{array}{c}\text { Total Non- } \\
\text { res }\end{array}$ & 98 & 210 & 98 & 162 & 160 & 159 & 158 & $-0.5 \%$ \\
\hline $\begin{array}{c}\text { Total } \\
\text { Lower }\end{array}$ & & 386 & & 368 & 380 & 382 & 384 & $0.8 \%$ \\
\hline $\begin{array}{c}\text { Total } \\
\text { Upper }\end{array}$ & & 953 & & 964 & 969 & 986 & 1,001 & $0.8 \%$ \\
\hline $\begin{array}{c}\text { Total Grad } \\
\text { I }\end{array}$ & & 975 & & 1,016 & 986 & 987 & 988 & $-0.6 \%$ \\
\hline $\begin{array}{c}\text { Total Grad } \\
\text { II }\end{array}$ & & 295 & & 273 & 286 & 293 & 294 & $1.5 \%$ \\
\hline Total FTE & 1,512 & 2,609 & 1,512 & 2,621 & 2,621 & 2,648 & 2,667 & $0.3 \%$ \\
\hline
\end{tabular}


For the sum of the remaining physical locations with fewer than 150 current or planned State-fundable FTE enrollments.

SITE: REMAINING PHYSICAL LOCATIONS See Appendices: USF; USF St. Petersburg; USF Sarasota-Manatee; USF Polytechnic

\begin{tabular}{|c|c|c|c|c|c|c|}
\hline & Estimated & Estimated & Estimated & Estimated & Estimated & 5-Year \\
\hline FTE & 2010-11 & 2011-12 & 2012-13 & 2014-15 & 2016-17 & $\begin{array}{c}\text { Average } \\
\text { Annual } \\
\text { Growth Rate }\end{array}$ \\
\hline Lower & & & & & & \\
\hline Upper & & & & & & \\
\hline Grad I & & & & & & \\
\hline Grad II & & & & & & \\
\hline Total & & & & & & \\
\hline
\end{tabular}

For the sum of current or planned State-fundable FTE enrollments not served at a physical location.

SITE: VIRTUAL INSTRUCTION / DISTANCE LEARNING

\begin{tabular}{|c|c|c|c|c|c|c|}
\hline & Estimated & Estimated & Estimated & Estimated & Estimated & $\begin{array}{c}\text { 5-Year } \\
\text { Projected } \\
\text { Average } \\
\text { Annual } \\
\text { Growth Rate }\end{array}$ \\
\hline FTE & $\mathbf{2 0 1 0 - 1 1}$ & $\mathbf{2 0 1 1 - 1 2}$ & $\mathbf{2 0 1 2 - 1 3}$ & $\mathbf{2 0 1 4 - 1 5}$ & $\mathbf{2 0 1 6 - 1 7}$ & $3 \%$ \\
\hline Lower & 1,099 & 1,132 & 1,167 & 1,237 & 1,313 & $3 \%$ \\
\hline Upper & 2,047 & 2,039 & 2,059 & 2,214 & 2,339 & $3 \%$ \\
\hline Grad I & 516 & 531 & 547 & 580 & 616 & $3 \%$ \\
\hline Total & 191 & 197 & 203 & 215 & 228 & $3 \%$ \\
\hline
\end{tabular}


Primary Institutional Goals/Metrics for the Next One to Three Years (In the context of the institutional strategic plan and vision, as well as System priorities, present three (3) to five (5) goals on which university effort will be focused in the next one to three years. Describe each goal, including whether the goal is new or continuing, the strategies for achieving that goal, the timeline and metrics by which success will be measured, expected outcomes, and assumptions, including financial, upon which the projected outcomes are predicated.) Each university is asked to include one goal associated with improved

baccalaureate retention and graduation (e.g., improved first-year retention; reduce attainment gaps for underrepresented groups; improve graduation rates for AA transfers; etc.).

\begin{tabular}{|c|c|c|c|c|c|c|c|c|c|}
\hline \multicolumn{3}{|c|}{$\begin{array}{c}\text { Institutional Goal } \\
\text { [Indicate whether NEW or CONTINUING] }\end{array}$} & \multicolumn{3}{|c|}{ Implementation Strategies } & \multicolumn{4}{|c|}{ Metric(s)/Timeline/Expected Outcomes } \\
\hline \multicolumn{3}{|c|}{$\begin{array}{l}\text { \#1 (Required) - IMPROVE } \\
\text { BACCALAUREATE RETENTION AND } \\
\text { GRADUATION } \\
\text { (Continuing) } \\
\text { The USF System is taking a comprehensive } \\
\text { approach to enhancing undergraduate } \\
\text { education to improve retention and } \\
\text { graduation rates. }\end{array}$} & \multicolumn{3}{|c|}{$\begin{array}{l}\text { - Implement state-of-the-art } \\
\text { degree audit system } \\
\text { (DegreeWorks) to promote } \\
\text { timely progression. } \\
\text { - Increase undergraduate } \\
\text { enrollment at the regional } \\
\text { institutions, } \\
\text { - Significant investments in } \\
\text { programs and services to help } \\
\text { students meet their fullest } \\
\text { potential. } \\
\text { - Increase number of academic } \\
\text { advisors, } \\
\text { - Expand tutoring services, } \\
\text { - Encourage undergraduate } \\
\text { research, } \\
\text { - Improve new student } \\
\text { orientation, remodel residence } \\
\text { halls, and update policies. } \\
\text { - Continue to improve on- } \\
\text { campus experience for students. }\end{array}$} & \multicolumn{4}{|c|}{$\begin{array}{l}\text { Metrics: } \\
\text { The USF System continues to monitor its retention and } \\
\text { graduation rate } \\
\text { (http://www.ods.usf.edu/Plans/PPA/matrix.htm). } \\
\text { - Monitor its investments in programs and services to } \\
\text { - } \quad \text { Melp students meet their fullest potential. } \\
\text { - Maintain number of academic advisors. } \\
\text { - Monitor tutoring services. } \\
\text { - Document undergraduate research. } \\
\text { - review student orientation practices. } \\
\text { Timeline: } \\
\text { This is an ongoing initiative, but improvements in } \\
\text { graduation rates are expected each year. } \\
\text { Expected outcomes: } \\
\text { Six year FTIC graduation rate of } 55 \% \text { in three years. } \\
\text { Three, four and five year graduation rates for transfer } \\
\text { students of } 55 \% \text { to } 60 \% \text { in three years. } \\
\text { Higher retention rates at all member institutions. }\end{array}$} \\
\hline \multicolumn{4}{|c|}{ Proposed Funding Source: $2011-12$} & \multicolumn{6}{|c|}{ Proposed Funding Source: $2012-13$} \\
\hline $\begin{array}{l}\text { State/ Tuition } \\
\text { Revenue (est.) }\end{array}$ & $\begin{array}{c}\text { Other } \\
\text { (Identify } \\
\text { Revenue } \\
\text { Source - } \\
\text { e.g., } \\
\text { Private) }\end{array}$ & $\begin{array}{l}\text { Undergrad. } \\
\text { Tuition } \\
\text { Differential } \\
\text { Revenue (est.) }\end{array}$ & $\begin{array}{c}\text { Total } \\
\text { from } \\
2011-12\end{array}$ & $\begin{array}{l}\text { Undergrad. } \\
\text { Tuition } \\
\text { Differential } \\
\text { Revenue } \\
\text { (est.) }\end{array}$ & $\begin{array}{l}\text { Legislative } \\
\text { Budget } \\
\text { Request } \\
\text { (State } \\
\text { Funds) }\end{array}$ & $\begin{array}{l}\text { State/ Tuition } \\
\text { Revenue (est.) }\end{array}$ & $\begin{array}{c}\text { Other } \\
\text { (Identify } \\
\text { Revenue } \\
\text { Source - } \\
\text { e.g., } \\
\text { Private) }\end{array}$ & $\begin{array}{l}\text { Total } \\
\text { from } \\
2012-13\end{array}$ & $\begin{array}{c}2012-13 \text { to } 2016-17 \\
\text { PECO/Courtelis } \\
\text { Request }\end{array}$ \\
\hline $0.143 \mathrm{M}$ & & $5.50 \mathrm{M}$ & $5.65 \mathrm{M}$ & $10.78 \mathrm{M}$ & $8.20 \mathrm{M}$ & $0.143 \mathrm{M}$ & & $19.12 \mathrm{M}$ & \\
\hline
\end{tabular}




\begin{tabular}{|c|c|c|c|c|c|c|c|c|c|}
\hline \multicolumn{3}{|c|}{$\begin{array}{c}\text { Institutional Goal } \\
\text { [Indicate whether NEW or CONTINUING] }\end{array}$} & \multicolumn{3}{|c|}{ Implementation Strategies } & \multicolumn{4}{|c|}{ Expected Outcomes/Metric(s)/Timeline } \\
\hline \multicolumn{3}{|c|}{$\begin{array}{l}\text { \#2 (Required) - Academic Excellence, } \\
\text { Student Access, and Student Success } \\
\text { (Continuing/New Elements): } \\
\text { The USF System is committed to academic } \\
\text { excellence, student access and student success }\end{array}$} & \multicolumn{3}{|c|}{$\begin{array}{l}\text { The USF System will: } \\
\text { - } \quad \text { develop an array of academic } \\
\text { programs that are recognized } \\
\text { regionally and nationally for } \\
\text { their rigor, intellectual } \\
\text { challenge, and high } \\
\text { expectations; } \\
\text { - } \quad \text { increase access to programs to } \\
\text { qualified and diverse students; } \\
\text { - } \quad \text { promote student learning and } \\
\text { success } \\
\text { - support activities that improve } \\
\text { retention and graduation rates, } \\
\text { employment and admission to } \\
\text { graduate or professional } \\
\text { schools. } \\
\text { The USF System is seeking to lower } \\
\text { average student debt loads, and } \\
\text { raise student satisfaction in } \\
\text { comparison with peer institutions. }\end{array}$} & \multicolumn{4}{|c|}{$\begin{array}{l}\text { Expected outcomes: } \\
\text { The USF System will continue to increase access to } \\
\text { programs to qualified and diverse students and promote } \\
\text { student learning and success by supporting activities that } \\
\text { improve retention and graduation rates, and support } \\
\text { employment and admission to graduate or professional } \\
\text { schools. The USF System will raise academic standards } \\
\text { across its member institutions with greater opportunities } \\
\text { and additional access for students, and increased } \\
\text { institutional efficiencies. } \\
\text { Metrics: } \\
\text { The USF System will monitor: } \\
\text { - Academic programs. } \\
\text { - Student access. } \\
\text { - Improvements in students' performances. } \\
\text { - Graduation rates. } \\
\text { - Employment opportunities. } \\
\text { - Graduate and professional school access. } \\
\text { Timeline: } \\
\text { This is an ongoing initiative with continuous quality } \\
\text { improvement expectations. }\end{array}$} \\
\hline \multicolumn{4}{|c|}{ Proposed Funding Source: $2011-12$} & \multicolumn{6}{|c|}{ Proposed Funding Source: $2012-13$} \\
\hline $\begin{array}{l}\text { State/ Tuition } \\
\text { Revenue (est.) }\end{array}$ & $\begin{array}{c}\text { Other } \\
\text { (Identify } \\
\text { Revenue } \\
\text { Source - } \\
\text { e.g., } \\
\text { Private) } \\
\end{array}$ & $\begin{array}{l}\text { Undergrad. } \\
\text { Tuition } \\
\text { Differential } \\
\text { Revenue (est.) }\end{array}$ & $\begin{array}{l}\text { Total } \\
\text { from } \\
\text { 2011-12 }\end{array}$ & $\begin{array}{l}\text { Undergrad } \\
\text { Tuition } \\
\text { Differential } \\
\text { Revenue } \\
\text { (est.) } \\
\end{array}$ & $\begin{array}{c}\text { Legislative } \\
\text { Budget } \\
\text { Request } \\
\text { (State } \\
\text { Funds) } \\
\end{array}$ & $\begin{array}{l}\text { State/ Tuition } \\
\text { Revenue (est.) }\end{array}$ & $\begin{array}{c}\text { Other } \\
\text { (Identify } \\
\text { Revenue } \\
\text { Source - } \\
\text { e.g., } \\
\text { Private) } \\
\end{array}$ & $\begin{array}{l}\text { Total } \\
\text { from } \\
2012-13\end{array}$ & $\begin{array}{c}\text { 2012-13 to 2016-17 } \\
\text { PECO/ Courtelis } \\
\text { Request }\end{array}$ \\
\hline $0.01 \mathrm{M}$ & $0.12 \mathrm{M}$ & - & $0.12 \mathrm{M}$ & $0.02 \mathrm{M}$ & $7.41 \mathrm{M}$ & $0.33 \mathrm{M}$ & $0.12 \mathrm{M}$ & $7.88 \mathrm{M}$ & \\
\hline
\end{tabular}




\begin{tabular}{|c|c|c|c|c|c|c|c|c|c|}
\hline \multicolumn{3}{|c|}{$\begin{array}{c}\text { Institutional Goal } \\
\text { [Indicate whether NEW or CONTINUING] }\end{array}$} & \multicolumn{3}{|c|}{ Implementation Strategies } & \multicolumn{4}{|c|}{ Expected Outcomes/Metric(s)/Timeline } \\
\hline \multicolumn{3}{|c|}{$\begin{array}{l}\text { \#3 (Required) - Impactful Research, } \\
\text { Economic Leadership and Community } \\
\text { Engagement (Continuing/New Elements): } \\
\text { The USF System will continue to challenge its } \\
\text { institutions to increase their levels of } \\
\text { community engagement by sharing best } \\
\text { practices; two members (USF and USF St. } \\
\text { Petersburg) have been designated Carnegie } \\
\text { “Community Engaged” institutions. }\end{array}$} & \multicolumn{3}{|c|}{$\begin{array}{l}\text { The USF System will continue to } \\
\text { coordinate and promote: } \\
\text { - } \quad \text { Research and innovation by } \\
\text { providing information and } \\
\text { services creating synergies } \\
\text { among faculty across the } \\
\text { System and fostering external } \\
\text { partnerships. } \\
\text { - Synergies among its institutions } \\
\text { to become a major economic } \\
\text { engine for the region and the } \\
\text { state. } \\
\text { - Increased production of } \\
\text { graduates in high demand } \\
\text { fields. } \\
\text { Increased applied research } \\
\text { supported by the private sector; } \\
\text { and } \\
\text { Incubation of new companies, } \\
\text { work with agencies, and } \\
\text { develop occupation } \\
\text { opportunities. }\end{array}$} & \multicolumn{4}{|c|}{$\begin{array}{l}\text { Expected outcomes: } \\
\text { The USF System will make a significant impact on the } \\
\text { economic opportunities for students and for the west- } \\
\text { central Florida region through its educational and } \\
\text { intellectual capacity. } \\
\text { Metrics: } \\
\text { USF System will monitor: } \\
\text { - Synergies among its institutions. } \\
\text { - Number of graduate students. } \\
\text { - Applied research endeavors. } \\
\text { - Private-public partnerships. } \\
\text { - New start-up companies. } \\
\text { - Community engagement activities. } \\
\text { Timeline: } \\
\text { This is an ongoing initiative with continuous quality } \\
\text { improvement expected. } \\
\text { The USF System will cultivate research/ development that } \\
\text { strengthens Florida's economy, provides new employment } \\
\text { opportunities, and contributes to global solutions. }\end{array}$} \\
\hline \multicolumn{4}{|c|}{ Proposed Funding Source: $2011-12$} & \multicolumn{6}{|c|}{ Proposed Funding Source: $2012-13$} \\
\hline $\begin{array}{l}\text { State/ Tuition } \\
\text { Revenue (est.) }\end{array}$ & $\begin{array}{c}\text { Other } \\
\text { (Identify } \\
\text { Revenue } \\
\text { Source - } \\
\text { e.g.; } \\
\text { Private) } \\
\end{array}$ & $\begin{array}{c}\text { Undergrad } \\
\text { Tuition } \\
\text { Differential } \\
\text { Revenue (est.) }\end{array}$ & $\begin{array}{c}\text { Total } \\
\text { from } \\
2011-12\end{array}$ & $\begin{array}{l}\text { Undergrad } \\
\text { Tuition } \\
\text { Differential } \\
\text { Revenue } \\
\text { (est.) }\end{array}$ & $\begin{array}{l}\text { Legislative } \\
\text { Budget } \\
\text { Request } \\
\text { (State } \\
\text { Funds) }\end{array}$ & $\begin{array}{l}\text { State/ Tuition } \\
\text { Revenue (est.) }\end{array}$ & $\begin{array}{c}\text { Other } \\
\text { (Identify } \\
\text { Revenue } \\
\text { Source - } \\
\text { e.g., } \\
\text { Private) } \\
\end{array}$ & $\begin{array}{l}\text { Total } \\
\text { from } \\
2012-13\end{array}$ & $\begin{array}{c}\text { 2012-13 to 2016-17 } \\
\text { PECO/Courtelis } \\
\text { Request }\end{array}$ \\
\hline $2.96 \mathrm{M}$ & $1.70 \mathrm{M}$ & & $4.66 \mathrm{M}$ & & $2.23 \mathrm{M}$ & $2.00 \mathrm{M}$ & $1.45 \mathrm{M}$ & $5.68 \mathrm{M}$ & \\
\hline
\end{tabular}


OPTIONAL: Universities may add one or two additional goals.

\begin{tabular}{|c|c|c|c|c|c|c|c|c|c|}
\hline \multicolumn{3}{|c|}{$\begin{array}{c}\text { Institutional Goal } \\
\text { [Indicate whether NEW or } \\
\text { CONTINUING] }\end{array}$} & \multicolumn{3}{|c|}{ Implementation Strategies } & \multicolumn{4}{|c|}{ Expected Outcomes/Metric(s)/Timeline } \\
\hline \multicolumn{3}{|c|}{$\begin{array}{l}\text { \#4 (Optional) - Increased Academic and } \\
\text { Administrative Collaborations } \\
\text { (Continuing/New Elements): } \\
\text { The USF System will continue to provide } \\
\text { leadership to increase academic and } \\
\text { administrative collaborations among its } \\
\text { institutions. }\end{array}$} & \multicolumn{3}{|c|}{$\begin{array}{l}\text { - Establishing opportunities for } \\
\text { students to enter graduate and } \\
\text { professional programs across the USF } \\
\text { System. } \\
\text { - Creating joint degree programs and } \\
\text { innovative cross-institution curricula. } \\
\text { - Promoting joint research activities } \\
\text { and other faculty development } \\
\text { opportunities. } \\
\text { - Improving the efficiency, } \\
\text { effectiveness, and functionality of } \\
\text { System-wide administrative } \\
\text { processes, systems, and technologies. } \\
\text { Continuously improving USF System } \\
\text { services, to increase user satisfaction. }\end{array}$} & \multicolumn{4}{|c|}{$\begin{array}{l}\text { Expected outcomes: } \\
\text { USF System will create new and innovative academic } \\
\text { programs, joint academic ventures and even greater } \\
\text { efficiencies amongst its member institutions. } \\
\text { Monitor: } \\
\text { The USF System will continue to monitor: } \\
\text { - Graduate and professional programs. } \\
\text { - Number of joint degree programs. } \\
\text { - Diversity of undergraduate programs. } \\
\text { - Research activity among member institutions. } \\
\text { - Student satisfaction. } \\
\text { Timeline: } \\
\text { This is an ongoing initiative with continuous quality } \\
\text { improvement expected. }\end{array}$} \\
\hline \multicolumn{4}{|c|}{ Proposed Funding Source: $2011-12$} & \multicolumn{6}{|c|}{ Proposed Funding Source: $2012-13$} \\
\hline $\begin{array}{l}\text { State/ Tuition } \\
\text { Revenue (est.) }\end{array}$ & $\begin{array}{c}\text { Other } \\
\text { (Identify } \\
\text { Revenue } \\
\text { Source - e.g., } \\
\text { Private) } \\
\end{array}$ & $\begin{array}{c}\text { Undergrad } \\
\text { Tuition } \\
\text { Differential } \\
\text { Revenue } \\
\text { (est.) } \\
\end{array}$ & $\begin{array}{l}\text { Total from } \\
\text { 2011-12 }\end{array}$ & $\begin{array}{c}\text { Undergrad } \\
\text { Tuition } \\
\text { Differential } \\
\text { Revenue } \\
\text { (est.) } \\
\end{array}$ & $\begin{array}{c}\text { Legislative } \\
\text { Budget } \\
\text { Request } \\
\text { (State Funds) }\end{array}$ & $\begin{array}{l}\text { State/ } \\
\text { Tuition } \\
\text { Revenue } \\
\text { (est.) }\end{array}$ & $\begin{array}{c}\text { Other } \\
\text { (Identify } \\
\text { Revenue } \\
\text { Source - } \\
\text { e.g., Private) }\end{array}$ & $\begin{array}{c}\text { Total from } \\
2012-13\end{array}$ & $\begin{array}{l}2012-13 \text { to } \\
2016-17 \\
\text { PECO/ } \\
\text { Courtelis } \\
\text { Request } \\
\end{array}$ \\
\hline $4.13 \mathrm{M}$ & - & $1.88 \mathrm{M}$ & $6.01 \mathrm{M}$ & $1.98 \mathrm{M}$ & $2.70 \mathrm{M}$ & $4.41 \mathrm{M}$ & - & $9.08 \mathrm{M}$ & \\
\hline & & & & & & & & & \\
\hline
\end{tabular}




\begin{tabular}{|c|c|c|c|c|c|c|c|c|c|}
\hline \multicolumn{3}{|c|}{$\begin{array}{c}\text { Institutional Goal } \\
\text { [Indicate whether NEW or } \\
\text { CONTINUING] }\end{array}$} & \multicolumn{3}{|c|}{ Implementation Strategies } & \multicolumn{4}{|c|}{ Expected Outcomes/Metric(s)/Timeline } \\
\hline \multicolumn{3}{|c|}{$\begin{array}{l}\text { \#5 (Optional) - Expanded and } \\
\text { Diversified Resources (Continuing/New } \\
\text { Elements): } \\
\text { The USF System continues to expand and } \\
\text { diversify its resource base to maintain } \\
\text { financial sustainability of its member } \\
\text { institutions and to assist them in meeting } \\
\text { their distinctive missions. }\end{array}$} & \multicolumn{3}{|c|}{$\begin{array}{l}\text { The USF System, in partnership with its } \\
\text { member institutions, continues to focus } \\
\text { on its: } \\
\text { - Endowment. } \\
\text { - Annual giving. } \\
\text { - Research grants and contracts. } \\
\text { - Revenues from auxiliaries and Direct } \\
\text { - Service Organizations. } \\
\text { - Overall efficiency. } \\
\text { The USF System, in partnership with the } \\
\text { leadership of all its institutions, continues } \\
\text { to work closely with the Board of } \\
\text { Governors to support the State University } \\
\text { System. }\end{array}$} & \multicolumn{4}{|c|}{$\begin{array}{l}\text { Expected outcomes: } \\
\text { Higher levels of external funding, private giving, and } \\
\text { more public-private partnerships, licenses and } \\
\text { patents that will foster greater employment } \\
\text { opportunities directed at New Florida initiatives. } \\
\text { Metrics: } \\
\text { - External funding. } \\
\text { - Endowment levels. } \\
\text { - Annual giving. } \\
\text { - Gesearch expenditures (Federal and total) } \\
\text { - Licenses, patents, start-ups. } \\
\text { Timeline: } \\
\text { This is an ongoing initiative with continuous quality } \\
\text { improvement expected. However, within the next } \\
\text { three years, the USF System will have moved } \\
\text { forward significantly in these areas. }\end{array}$} \\
\hline \multicolumn{4}{|c|}{ Proposed Funding Source: $2011-12$} & \multicolumn{6}{|c|}{ Proposed Funding Source: $2012-13$} \\
\hline $\begin{array}{l}\text { State/ Tuition } \\
\text { Revenue (est.) }\end{array}$ & $\begin{array}{c}\text { Other } \\
\text { (Identify } \\
\text { Revenue } \\
\text { Source - e.g., } \\
\text { Private) } \\
\end{array}$ & $\begin{array}{c}\text { Undergrad } \\
\text { Tuition } \\
\text { Differential } \\
\text { Revenue } \\
\text { (est.) } \\
\end{array}$ & $\begin{array}{l}\text { Total from } \\
\text { 2011-12 }\end{array}$ & $\begin{array}{c}\text { Undergrad } \\
\text { Tuition } \\
\text { Differential } \\
\text { Revenue } \\
\text { (est.) } \\
\end{array}$ & $\begin{array}{l}\text { Legislative } \\
\text { Budget } \\
\text { Request } \\
\text { (State Funds) }\end{array}$ & $\begin{array}{l}\text { State/ } \\
\text { Tuition } \\
\text { Revenue } \\
\text { (est.) }\end{array}$ & $\begin{array}{c}\text { Other } \\
\text { (Identify } \\
\text { Revenue } \\
\text { Source - } \\
\text { e.g., Private) } \\
\end{array}$ & $\begin{array}{l}\text { Total from } \\
2012-13\end{array}$ & $\begin{array}{c}2012-13 \text { to } \\
2016-17 \\
\text { PECO/ } \\
\text { Courtelis } \\
\text { Request } \\
\end{array}$ \\
\hline $0.07 \mathrm{M}$ & - & - & $0.07 \mathrm{M}$ & - & $2.00 \mathrm{M}$ & $0.06 \mathrm{M}$ & - & $2.06 \mathrm{M}$ & \\
\hline
\end{tabular}




\begin{tabular}{|c|c|c|c|c|c|c|c|c|c|c|}
\hline \multicolumn{11}{|c|}{ SUMMARY OF PROPOSED FUNDING FOR PRIMARY GOALS } \\
\hline \multicolumn{5}{|c|}{ Proposed Funding Source: $2011-12$} & \multicolumn{6}{|c|}{ Proposed Funding Source: $2012-13$} \\
\hline Goal \# & $\begin{array}{c}\text { State/ } \\
\text { Tuition } \\
\text { Revenue } \\
\text { (est.) }\end{array}$ & $\begin{array}{c}\text { Other } \\
\text { (Identify } \\
\text { Revenue } \\
\text { Source - } \\
\text { e.g., } \\
\text { Private) } \\
\end{array}$ & $\begin{array}{l}\text { Undergrad } \\
\text { Tuition } \\
\text { Differentia } \\
1 \text { Revenue } \\
\text { (est.) }\end{array}$ & $\begin{array}{c}\text { Total from } \\
2011-12\end{array}$ & $\begin{array}{c}\text { Undergrad } \\
\text { Tuition } \\
\text { Differential } \\
\text { Revenue } \\
\text { (est.) }\end{array}$ & $\begin{array}{l}\text { Legislative } \\
\text { Budget } \\
\text { Request } \\
\text { (State } \\
\text { Funds) }\end{array}$ & $\begin{array}{l}\text { State/ Tuition } \\
\text { Revenue } \\
\text { (est.) }\end{array}$ & $\begin{array}{c}\text { Other } \\
\text { (Identify } \\
\text { Revenue } \\
\text { Source - } \\
\text { e.g., } \\
\text { Private) } \\
\end{array}$ & $\begin{array}{l}\text { Total } \\
\text { from } \\
2012-13\end{array}$ & $\begin{array}{c}2012-13 \\
\text { to } 2016 \\
-17 \\
\text { PECO/ } \\
\text { Courtelis } \\
\text { Request } \\
\end{array}$ \\
\hline 1 & 142,750 & - & $5,502,981$ & $5,645,731$ & $10,778,621$ & $8,210,956$ & 142,750 & - & $19,132,327$ & \\
\hline 2 & 10,000 & 122,500 & - & 123,500 & 24,000 & $7,407,567$ & 330,000 & 122,500 & $7,875,067$ & \\
\hline 3 & $2,963,299$ & $1,700,000$ & - & $4,663,299$ & - & $2,225,000$ & $2,000,000$ & $1,450,000$ & $5,675,000$ & - \\
\hline 4 optional & $4,125,000$ & - & $1,881,304$ & $6,006,304$ & $1,975,369$ & $2,700,000$ & $4,405,000$ & - & $9,080,369$ & . \\
\hline 5 optional & 70,000 & - & - & 70,000 & - & $2,000,000$ & 60,000 & - & $2,060,000$ & \\
\hline Total & $7,311,049$ & $1,822,500$ & $7,384,285$ & $16,508,834$ & $12,777,990$ & $22,993,750$ & $6,937,750$ & $1,572,500$ & $43,822,763$ & \\
\hline
\end{tabular}




\section{0 - 2011 Tuition Differential Update}

Provide the following information for the 2010-2011 Academic Year.

Note: For details see individual campus data in appendices

2010-2011 - 70\% Initiatives (List the initiatives provided in the 2010-11 tuition differential request.)

Increase course offerings

Improve graduation rates

Increase the percentage of undergraduate students who are taught by faculty

Decrease student-faculty ratios

\section{University Update on Each Initiative}

Fall 2010 there were 263 additional sections taught compared to Fall 2009 which has increased accessibility.

There has been a notable change graduation rates; they were $48.1 \%$ in 2009 and have risen three points to $51.6 \%$ in 2010 .

There has been an increase in the percentage of undergraduates taught by faculty, in accessibility, and in the number of degrees awarded.

The student population increased over the last five years, even though resources were invested in faculty so the student to faculty ratio has remained relatively stable at 27:1.

\section{Additional Detail, Where Applicable:}

Total Number of Faculty Hired or Retained (funded by tuition differential):

88

Total Number of Advisors Hired or Retained (funded by tuition differential):

Total Number of Course Sections Added or Saved (funded by tuition differential):

2010-2011 - 30\% Initiatives (list the initiatives provided in the 2010-11 tuition differential request)

We will continue to target our need based grant awards to students who are paying the differential charges.

Because we continue to experience an increase in FAFSA filers who have need, the differential revenue will prevent dilution of the need based funds that are being awarded to an increasing number of students.

We will continue to target our need based grant awards to students who are paying the differential charges.

\section{Additional Information (estimates as of April 30, 2011):}

Unduplicated Count of Students Receiving at least one Tuition Differential-Funded Award: University Update on Each Initiative

Update to total expenditures: $\$ 3,203,325$

Update to total expenditures: $\$ 1,165,221$

Update to total expenditures: \$3,203,325
\$ Mean (per student receiving an award) of Tuition Differential-Funded Awards:

\$ Minimum (per student receiving an award) of Tuition Differential-Funded Awards:

\$ Maximum (per student receiving an award) of Tuition Differential-Funded Awards:
3696

Varies by member institution (\$757 to $\$ 1187$ )

Varies by member institution ( $\$ 160$ to $\$ 255$ )

Varies by member institution ( $\$ 1,000$ to $\$ 2,882)$ 


\section{Fall 2011 Request for an Increased Tuition Differential Fee}

\section{University: USF System Note: For details see individual campus data in appendices}

\section{Effective Date}

University Board of Trustees Approval Date:

June 2011

Campus or Center Location

Campus or Center Location to which the Tuition

Differential fee will apply (If the entire university, indicate as such):

Undergraduate Course(s)

Course(s). (If the tuition differential fee applies to

all university undergraduate courses, indicate as

such. If not, also provide a rationale for the

differentiation among courses):

Current and Proposed Increase in the Tuition Differential Fee

Current Undergraduate Tuition Differential per credit hour:

Percentage tuition differential fee increase

(calculated as a percentage of the sum of base

tuition plus tuition differential):

$\$$ Increase in tuition differential per credit hour:

$\$$ Increase in tuition differential for 30 credit hours:

USF System

USF System

Projected Differential Revenue Generated and Intended Uses

Incremental differential fee revenue generated in

2011-12 (projected):

Total differential fee revenue generated in 2011-12

(projected):

Varies by member institution ( $\$ 12.8$ to $\$ 39.65)$

Varies by member institution ( $\$ 16.27$ to $\$ 17.65)$

Varies by Campus ( $\$ 488.1$ to $\$ 529.5$ )

Varies by member institution

Varies by member institution 


\section{STATE UNIVERSITY SYSTEM OF FLORIDA \\ Tuition Differential Collections, Expenditures, and Available Balances \\ University of South Florida - System \\ Fiscal Year 2010-2011 and 2011-12}

University Tuition Differential

Budget Entity: 48900100 (Educational \& General)

SF/Fund: 2164xxx (Student and Other Fees Trust Fund)

\begin{tabular}{|c|c|c|c|c|}
\hline \multirow[b]{2}{*}{ Balance Forward from Prior Periods } & \multicolumn{2}{|c|}{$\begin{array}{c}\text { Estimated Actual }^{*} \\
2010-11 \\
\end{array}$} & \multicolumn{2}{|c|}{$\begin{array}{c}\text { Estimated } \\
2011-12\end{array}$} \\
\hline & & & & \\
\hline Balance Forward & $\$$ & 396,824 & $\$$ & $1,198,446$ \\
\hline Less: Prior-Year Encumbrances & & - & & - \\
\hline Beginning Balance Available: & $\$$ & 396,824 & $\$$ & $1,198,446$ \\
\hline \multicolumn{5}{|l|}{ Receipts / Revenues } \\
\hline Tuition Differential Collections & $\$$ & $14,536,658$ & $\$$ & $22,924,331$ \\
\hline Interest Revenue - Current Year & & 22,985 & & 36,287 \\
\hline Interest Revenue - From Carryforward Balance & & 537 & & 2,128 \\
\hline Total Receipts / Revenues: & $\$$ & $14,560,180$ & $\$$ & $22,962,746$ \\
\hline \multicolumn{5}{|l|}{ Expenditures } \\
\hline Salaries \& Benefits & $\$$ & $9,003,003$ & $\$$ & $15,042,441$ \\
\hline Other Personal Services & & 248,457 & & 959,917 \\
\hline Expenses & & 16,400 & & - \\
\hline Operating Capital Outlay & & - & & - \\
\hline Student Financial Assistance & & $4,378,459$ & & $6,887,140$ \\
\hline Expended From Carryforward Balance & & 112,239 & & $1,022,359$ \\
\hline${ }^{* *}$ Other Category Expenditures & & - & & - \\
\hline Total Expenditures: & $\$$ & $13,758,558$ & $\$$ & $23,911,857$ \\
\hline Ending Balance Available: & $\$$ & $1,198,446$ & $\$$ & 249,335 \\
\hline
\end{tabular}

*Since the 2010-11 year has not been completed, provide an estimated actual.

**Provide details for "Other Categories" used. 


\section{University of South Florida - Main Campus}

\begin{tabular}{|c|c|c|c|c|c|c|c|}
\hline Undergraduate Students & \multicolumn{3}{|c|}{ |-----------------Actual--------------- } & \multicolumn{4}{|c|}{ 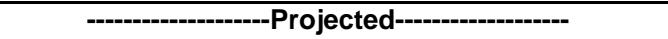 } \\
\hline \multicolumn{8}{|l|}{ Tuition: } \\
\hline Base Tuition - (0\% projected legislative increase) & $\$ 82.03$ & $\$ 88.59$ & $\$ 95.67$ & $\$ 103.32$ & $\$ 103.32$ & $\$ 103.32$ & $\$ 103.32$ \\
\hline Total Base Tuition and Differential & $\$ 88.99$ & $\$ 102.33$ & $\$ 117.67$ & $\$ 135.32$ & $\$ 155.62$ & $\$ 178.96$ & $\$ 205.80$ \\
\hline$\%$ Change & & $15.0 \%$ & $15.0 \%$ & $15.0 \%$ & $15.0 \%$ & $15.0 \%$ & $15.0 \%$ \\
\hline Building/Capital Improvement ${ }^{2}$ & $\$ 4.76$ & $\$ 4.76$ & $\$ 4.76$ & $\$ 4.76$ & $\$ 4.76$ & $\$ 4.76$ & $\$ 4.76$ \\
\hline Activity \& Service & $\$ 8.79$ & $\$ 9.31$ & $\$ 11.28$ & $\$ 11.28$ & $\$ 11.51$ & $\$ 11.74$ & $\$ 11.97$ \\
\hline Health & $\$ 7.91$ & $\$ 8.60$ & $\$ 9.30$ & $\$ 9.73$ & $\$ 9.92$ & $\$ 10.12$ & $\$ 10.33$ \\
\hline Athletic & $\$ 11.50$ & $\$ 11.76$ & $\$ 13.73$ & $\$ 14.15$ & $\$ 14.43$ & $\$ 14.72$ & $\$ 15.02$ \\
\hline Transportation Access & $\$ 3.00$ & $\$ 3.00$ & $\$ 3.00$ & $\$ 3.00$ & $\$ 3.00$ & $\$ 3.00$ & $\$ 3.00$ \\
\hline Technology ${ }^{1}$ & & $\$ 4.42$ & $\$ 4.78$ & $\$ 5.16$ & $\$ 5.16$ & $\$ 5.16$ & $\$ 5.16$ \\
\hline \multicolumn{8}{|l|}{ Fees (block per term): } \\
\hline $\begin{array}{l}\text { Activity \& Service } \\
\text { Health }\end{array}$ & $\$ 7.00$ & $\$ 7.00$ & $\$ 7.00$ & $\$ 7.00$ & $\$ 7.00$ & $\$ 7.00$ & $\$ 7.00$ \\
\hline $\begin{array}{l}\text { Athletic } \\
\text { Transportation Access }\end{array}$ & $\$ 10.00$ & $\$ 10.00$ & $\$ 10.00$ & $\$ 10.00$ & $\$ 10.00$ & $\$ 10.00$ & $\$ 10.00$ \\
\hline Other (list): Marshall Center & $\$ 20.00$ & $\$ 20.00$ & $\$ 20.00$ & $\$ 20.00$ & $\$ 20.00$ & $\$ 20.00$ & $\$ 20.00$ \\
\hline Total Block Fees per term & $\$ 37.00$ & $\$ 37.00$ & $\$ 37.00$ & $\$ 37.00$ & $\$ 37.00$ & $\$ 37.00$ & $\$ 37.00$ \\
\hline$\%$ Change & & $0.0 \%$ & $0.0 \%$ & $0.0 \%$ & $0.0 \%$ & $0.0 \%$ & $0.0 \%$ \\
\hline Total Tuition and Fees for $\mathbf{3 0}$ credit hours & $\$ 3,990.50$ & $\$ 4,577.00$ & $\$ 5,198.00$ & $\$ 5,805.80$ & $\$ 6,435.84$ & $\$ 7,157.63$ & $\$ 7,984.91$ \\
\hline$\%$ Change & & $14.7 \%$ & $13.6 \%$ & $11.7 \%$ & $10.9 \%$ & $11.2 \%$ & $11.6 \%$ \\
\hline \multicolumn{8}{|l|}{ Out-of-State Fees } \\
\hline \% Change & & $8.3 \%$ & $2.9 \%$ & $4.0 \%$ & $4.0 \%$ & $4.0 \%$ & $4.0 \%$ \\
\hline
\end{tabular}

\footnotetext{
${ }^{1}$ can be no more than $5 \%$ of tuition. $\quad{ }^{3}$ can be no more than $5 \%$ of tuition and the out-of-state fee.
}

${ }^{2}$ capped in statute. 
University of South Florida - St. Petersburg

\begin{tabular}{|c|c|c|c|c|c|c|c|}
\hline Undergraduate Students & \multicolumn{3}{|c|}{-----------------Actual--------------- } & \multicolumn{4}{|c|}{ 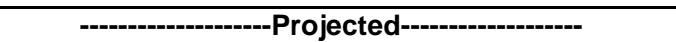 } \\
\hline \multicolumn{8}{|l|}{ Tuition: } \\
\hline Tuition Differential (no more than 15\%) & & $\$ 5.74$ & $\$ 12.80$ & $\$ 21.42$ & $\$ 40.13$ & $\$ 61.65$ & $\$ 86.40$ \\
\hline Total Base Tuition and Differential & $\$ 82.03$ & $\$ 94.33$ & $\$ 108.47$ & $\$ 124.74$ & $\$ 143.46$ & $\$ 164.97$ & $\$ 189.72$ \\
\hline \% Change & & $15.0 \%$ & $15.0 \%$ & $15.0 \%$ & $15.0 \%$ & $15.0 \%$ & $15.0 \%$ \\
\hline \multicolumn{8}{|l|}{ Fees (per credit hour): } \\
\hline Building/Capital Improvement ${ }^{2}$ & $\$ 4.76$ & $\$ 4.76$ & $\$ 4.76$ & $\$ 4.76$ & $\$ 4.76$ & $\$ 4.76$ & $\$ 4.76$ \\
\hline Activity \& Service & $\$ 9.76$ & $\$ 10.40$ & $\$ 24.80$ & $\$ 24.80$ & $\$ 24.80$ & $\$ 24.80$ & $\$ 24.80$ \\
\hline Health & $\$ 0.60$ & $\$ 0.60$ & $\$ 1.20$ & $\$ 2.64$ & $\$ 2.77$ & $\$ 2.91$ & $\$ 3.06$ \\
\hline Athletic & $\$ 2.25$ & $\$ 2.25$ & $\$ 2.45$ & $\$ 2.45$ & $\$ 2.45$ & $\$ 2.45$ & $\$ 2.45$ \\
\hline Transportation Access & $\$ 2.25$ & $\$ 2.25$ & $\$ 2.25$ & $\$ 2.25$ & $\$ 2.25$ & $\$ 2.25$ & $\$ 2.25$ \\
\hline Technology ${ }^{1}$ & & $\$ 4.42$ & $\$ 4.78$ & $\$ 5.16$ & $\$ 5.16$ & $\$ 5.16$ & $\$ 5.16$ \\
\hline \multicolumn{8}{|l|}{ Fees (block per term): } \\
\hline \multicolumn{8}{|l|}{ Activity \& Service } \\
\hline \multicolumn{8}{|l|}{ Health } \\
\hline Athletic & $\$ 5.00$ & $\$ 5.00$ & $\$ 5.00$ & $\$ 5.00$ & $\$ 5.00$ & $\$ 5.00$ & $\$ 5.00$ \\
\hline \multicolumn{8}{|l|}{ Transportation Access } \\
\hline \multirow{2}{*}{$\begin{array}{r}\text { Total Block Fees per term } \\
\% \text { Change }\end{array}$} & $\$ 5.00$ & $\$ 5.00$ & $\$ 5.00$ & $\$ 5.00$ & $\$ 5.00$ & $\$ 5.00$ & $\$ 5.00$ \\
\hline & & $0.0 \%$ & $0.0 \%$ & $0.0 \%$ & $0.0 \%$ & $0.0 \%$ & $0.0 \%$ \\
\hline Total Tuition and Fees for 30 credit hours & $\$ 3,182.50$ & $\$ 3,712.90$ & $\$ 4,614.70$ & $\$ 5,198.91$ & $\$ 5,764.21$ & $\$ 6,413.92$ & $\$ 7,160.67$ \\
\hline$\%$ Change & & $16.7 \%$ & $24.3 \%$ & $12.7 \%$ & $10.9 \%$ & $11.3 \%$ & $11.6 \%$ \\
\hline \multicolumn{8}{|l|}{ Out-of-State Fees } \\
\hline
\end{tabular}

\footnotetext{
${ }^{1}$ can be no more than $5 \%$ of tuition.

${ }^{3}$ can be no more than $5 \%$ of tuition and the out-of-state fee.

${ }^{2}$ capped in statute.
} 


\section{University of South Florida - Sarasota/Manatee}

\begin{tabular}{|c|c|c|c|c|c|c|c|}
\hline \multirow{2}{*}{ Undergraduate Students } & \multicolumn{3}{|c|}{------------------Actual--------------- } & \multicolumn{4}{|c|}{-------------------Projected----------------- } \\
\hline & 2008-09 & 2009-10 & 2010-11 & 2011-12 & 2012-13 & 2013-14 & 2014-15 \\
\hline \multicolumn{8}{|l|}{ Tuition: } \\
\hline$\overline{\text { Base Tuition - (projected legislative increase) }}$ & $\$ 82.03$ & $\$ 88.59$ & $\$ 95.67$ & $\$ 103.32$ & $\$ 103.32$ & $\$ 103.32$ & $\$ 103.32$ \\
\hline Tuition Differential (no more than $15 \%$ ) & & $\$ 5.74$ & $\$ 12.80$ & $\$ 21.42$ & $\$ 40.13$ & $\$ 61.65$ & $\$ 86.40$ \\
\hline Total Base Tuition and Differential & $\$ 82.03$ & $\$ 94.33$ & $\$ 108.47$ & $\$ 124.74$ & $\$ 143.46$ & $\$ 164.97$ & $\$ 189.72$ \\
\hline \% Change & & $15.0 \%$ & $15.0 \%$ & $15.0 \%$ & $15.0 \%$ & $15.0 \%$ & $15.0 \%$ \\
\hline \multicolumn{8}{|l|}{ Fees (per credit hour): } \\
\hline Student Financial Aid ${ }^{1}$ & $\$ 4.10$ & $\$ 4.42$ & $\$ 4.78$ & $\$ 5.16$ & $\$ 5.16$ & $\$ 5.16$ & $\$ 5.16$ \\
\hline Building/Capital Improvement ${ }^{2}$ & $\$ 4.76$ & $\$ 4.76$ & $\$ 4.76$ & $\$ 4.76$ & $\$ 4.76$ & $\$ 4.76$ & $\$ 4.76$ \\
\hline Activity \& Service & $\$ 9.82$ & $\$ 9.82$ & $\$ 20.19$ & $\$ 21.00$ & $\$ 21.84$ & $\$ 22.71$ & $\$ 23.62$ \\
\hline Health & $\$ 2.00$ & $\$ 2.00$ & $\$ 4.03$ & $\$ 4.19$ & $\$ 4.36$ & $\$ 4.53$ & $\$ 4.71$ \\
\hline Athletic & $\$ 2.10$ & $\$ 2.10$ & $\$ 4.23$ & $\$ 4.40$ & $\$ 4.58$ & $\$ 4.76$ & $\$ 4.95$ \\
\hline \multicolumn{8}{|l|}{ Transportation Access } \\
\hline Technology $^{1}$ & & $\$ 4.42$ & $\$ 4.78$ & $\$ 5.16$ & $\$ 5.16$ & $\$ 5.16$ & $\$ 5.16$ \\
\hline \multicolumn{4}{|l|}{ Student Green Energy (New authority - campus optional) } & & $\$ 1.00$ & $\$ 1.00$ & $\$ 1.00$ \\
\hline Total Tuition and Fees per credit hour & $\$ 104.81$ & $\$ 121.85$ & $\$ 151.24$ & $\$ 169.41$ & $\$ 190.31$ & $\$ 213.06$ & $\$ 239.08$ \\
\hline$\%$ Change & & $16.3 \%$ & $24.1 \%$ & $12.0 \%$ & $12.3 \%$ & $12.0 \%$ & $12.2 \%$ \\
\hline \multicolumn{8}{|l|}{ Fees (block per term): } \\
\hline \multirow{2}{*}{\multicolumn{8}{|c|}{$\begin{array}{l}\text { Activity \& Service } \\
\text { Health }\end{array}$}} \\
\hline & & & & & & & \\
\hline Athletic & $\$ 5.00$ & $\$ 5.00$ & $\$ 5.00$ & $\$ 5.00$ & $\$ 5.00$ & $\$ 5.00$ & $\$ 5.00$ \\
\hline \multicolumn{8}{|l|}{ Transportation Access } \\
\hline \multirow[t]{2}{*}{ Total Block Fees per term } & $\$ 5.00$ & $\$ 5.00$ & $\$ 5.00$ & $\$ 5.00$ & $\$ 5.00$ & $\$ 5.00$ & $\$ 5.00$ \\
\hline & & $0.0 \%$ & $0.0 \%$ & $0.0 \%$ & $0.0 \%$ & $0.0 \%$ & $0.0 \%$ \\
\hline \multirow{2}{*}{$\begin{array}{l}\text { Total Tuition and Fees for } 30 \text { credit hours } \\
\% \text { Change }\end{array}$} & $\$ 3,154.30$ & $\$ 3,665.50$ & $\$ 4,547.20$ & $\$ 5,092.35$ & $\$ 5,719.20$ & $\$ 6,401.67$ & $\$ 7,182.46$ \\
\hline & & $16.2 \%$ & $24.1 \%$ & $12.0 \%$ & $12.3 \%$ & $11.9 \%$ & $12.2 \%$ \\
\hline \multicolumn{8}{|l|}{ Out-of-State Fees } \\
\hline \multirow{3}{*}{$\begin{array}{l}\text { Out-of-State Undergraduate Fee } \\
\text { Out-of-State Undergraduate Student Financial Aid }{ }^{3} \\
\text { Total per credit hour } \\
\qquad\end{array}$} & $\$ 403.72$ & $\$ 343.16$ & $\$ 343.16$ & $\$ 291.68$ & $\$ 291.68$ & $\$ 291.68$ & $\$ 291.68$ \\
\hline & $\$ 20.18$ & $\$ 17.15$ & $\$ 17.15$ & $\$ 14.58$ & $\$ 14.58$ & $\$ 14.58$ & $\$ 14.58$ \\
\hline & $\$ 423.90$ & $\$ 360.31$ & $\$ 360.31$ & $\$ 306.26$ & $\$ 306.26$ & $\$ 306.26$ & $\$ 306.26$ \\
\hline \multirow{3}{*}{$\begin{array}{l}\text { \% Change } \\
\text { Total Tuition and Fees for } 30 \text { Credit Hours } \\
\% \text { Change }\end{array}$} & & $-15 \%$ & $0 \%$ & $-15 \%$ & $0 \%$ & $0 \%$ & $0 \%$ \\
\hline & $\$ 15,871.30$ & $\$ 14,474.80$ & $\$ 15,356.50$ & $\$ 14,280.15$ & $\$ 14,907.00$ & $\$ 15,589.47$ & $\$ 16,370.26$ \\
\hline & & $-9 \%$ & $6 \%$ & $-7 \%$ & $4 \%$ & $5 \%$ & $5 \%$ \\
\hline \multirow[t]{2}{*}{ Housing/Dining } & $\mathrm{N} / \mathrm{A}$ & $\mathrm{N} / \mathrm{A}$ & $\mathrm{N} / \mathrm{A}$ & $\mathrm{N} / \mathrm{A}$ & $\mathrm{N} / \mathrm{A}$ & $\mathrm{N} / \mathrm{A}$ & N/A \\
\hline & & & & & & & \\
\hline
\end{tabular}

${ }^{1}$ can be no more than $5 \%$ of tuition. $\quad{ }^{3}$ can be no more than $5 \%$ of tuition and the out-of-state fee.

2 capped in statute. 
University of South Florida - Polytechnic

\begin{tabular}{|c|c|c|c|c|c|c|c|}
\hline \multirow{2}{*}{ Undergraduate Students } & \multicolumn{3}{|c|}{-----------------Actual--------------- } & \multicolumn{4}{|c|}{ 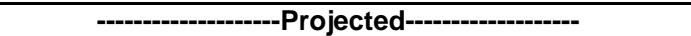 } \\
\hline & 2008-09 & 2009-10 & 2010-11 & 2011-12 & 2012-13 & 2013-14 & 2014-15 \\
\hline \multicolumn{8}{|l|}{ Tuition: } \\
\hline Base Tuition - (projected legislative increase) & $\$ 82.03$ & $\$ 88.59$ & $\$ 95.67$ & $\$ 103.32$ & $\$ 103.32$ & $\$ 103.32$ & $\$ 103.32$ \\
\hline Total Base Tuition and Differential & $\$ 82.03$ & $\$ 94.33$ & $\$ 108.47$ & $\$ 124.74$ & $\$ 143.46$ & $\$ 164.97$ & $\$ 189.72$ \\
\hline \% Change & & $15.0 \%$ & $15.0 \%$ & $15.0 \%$ & $15.0 \%$ & $15.0 \%$ & $15.0 \%$ \\
\hline Building/Capital Improvement ${ }^{2}$ & $\$ 4.76$ & $\$ 4.76$ & $\$ 4.76$ & $\$ 4.76$ & $\$ 4.76$ & $\$ 4.76$ & $\$ 4.76$ \\
\hline Activity \& Service & $\$ 5.76$ & $\$ 6.17$ & $\$ 24.35$ & $\$ 24.35$ & $\$ 24.35$ & $\$ 24.35$ & $\$ 24.35$ \\
\hline Health & & & & $\$ 1.44$ & $\$ 1.44$ & $\$ 1.44$ & $\$ 1.44$ \\
\hline \multirow{2}{*}{\multicolumn{8}{|c|}{ Transportation Access }} \\
\hline & & & & & & & \\
\hline Technology ${ }^{1}$ & & $\$ 4.42$ & $\$ 4.78$ & $\$ 5.16$ & $\$ 5.16$ & $\$ 5.16$ & $\$ 5.16$ \\
\hline \multicolumn{4}{|l|}{ Student Green Energy (New authority - campus optional) } & & $\$ 1.00$ & $\$ 1.00$ & $\$ 1.00$ \\
\hline \multicolumn{8}{|l|}{ Fees (block per term): } \\
\hline \multicolumn{8}{|l|}{ Activity \& Service } \\
\hline \multicolumn{8}{|l|}{ Health } \\
\hline Athletic & $\$ 5.00$ & $\$ 5.00$ & $\$ 5.00$ & $\$ 5.00$ & $\$ 5.00$ & $\$ 5.00$ & $\$ 5.00$ \\
\hline \multicolumn{8}{|l|}{ Transportation Access } \\
\hline \multirow{2}{*}{$\begin{array}{r}\text { Total Block Fees per term } \\
\% \text { Change }\end{array}$} & $\$ 5.00$ & $\$ 5.00$ & $\$ 5.00$ & $\$ 5.00$ & $\$ 5.00$ & $\$ 5.00$ & $\$ 5.00$ \\
\hline & & $0.0 \%$ & $0.0 \%$ & $0.0 \%$ & $0.0 \%$ & $0.0 \%$ & $0.0 \%$ \\
\hline \multirow{2}{*}{$\begin{array}{l}\text { Total Tuition and Fees for } 30 \text { credit hours } \\
\% \text { Change }\end{array}$} & $\$ 2,972.50$ & $\$ 3,496.00$ & $\$ 4,487.20$ & $\$ 5,041.41$ & $\$ 5,632.75$ & $\$ 6,278.30$ & $\$ 7,020.68$ \\
\hline & & $17.6 \%$ & $28.4 \%$ & $12.4 \%$ & $11.7 \%$ & $11.5 \%$ & $11.8 \%$ \\
\hline \multicolumn{8}{|l|}{ Out-of-State Fees } \\
\hline \multirow{2}{*}{$\begin{array}{l}\text { Out-of-State Undergraduate Fee } \\
\text { Out-of-State Undergraduate Student Financial Aid } \\
\text { Total per credit hour } \\
\qquad \\
\end{array}$} & $\$ 403.72$ & $\$ 343.16$ & $\$ 343.16$ & $\$ 291.68$ & $\$ 291.68$ & $\$ 291.68$ & $\$ 291.68$ \\
\hline & $\$ 20.18$ & $\$ 17.15$ & $\$ 17.15$ & $\$ 14.58$ & $\$ 14.58$ & $\$ 14.58$ & $\$ 14.58$ \\
\hline \multirow{3}{*}{$\begin{array}{l}\text { \% Change } \\
\text { Total Tuition and Fees for } 30 \text { Credit Hours } \\
\% \text { Change }\end{array}$} & & $-15 \%$ & $0 \%$ & $-15 \%$ & $0 \%$ & $0 \%$ & $0 \%$ \\
\hline & $\$ 15,689.50$ & $\$ 14,305.30$ & $\$ 15,296.50$ & $\$ 14,229.21$ & $\$ 14,820.55$ & $\$ 15,466.10$ & $\$ 16,208.48$ \\
\hline & & $-9 \%$ & $7 \%$ & $-7 \%$ & $4 \%$ & $4 \%$ & $5 \%$ \\
\hline \multirow[t]{2}{*}{ Housing/Dining } & $\mathrm{N} / \mathrm{A}$ & $\mathrm{N} / \mathrm{A}$ & N/A & N/A & \multirow[t]{2}{*}{ N/A } & \multirow[t]{2}{*}{ N/A } & \multirow[t]{2}{*}{ N/A } \\
\hline & & & & & & & \\
\hline
\end{tabular}

\footnotetext{
${ }^{1}$ can be no more than $5 \%$ of tuition.

${ }^{3}$ can be no more than $5 \%$ of tuition and the out-of-state fee.

${ }^{2}$ capped in statute.
} 


\section{University of South Florida 2012-13 Legislative Budget Request}

\begin{tabular}{|c|c|c|c|c|c|}
\hline $\begin{array}{l}\text { Priority } \\
\text { Number }\end{array}$ & Campus & Work Plan Issue Title / Other Issue & $\begin{array}{l}\text { Recurring } \\
\text { Funds }\end{array}$ & $\begin{array}{l}\text { Non- } \\
\text { recurring } \\
\text { Funds }\end{array}$ & Total Funds \\
\hline 1 & Tampa & $\begin{array}{l}\text { Program Access and Degree Production in } \\
\text { STEM Fields }\end{array}$ & $\$ 8,217,981$ & $\$ 0$ & $\$ 8,217,981$ \\
\hline 2 & Tampa & $\begin{array}{l}\text { Competitive PhD Student Recruitment in } \\
\text { STEM Fields }\end{array}$ & $\$ 3,047,806$ & $\$ 0$ & $\$ 3,047,806$ \\
\hline 3 & Tampa & Clinical Translational Sciences Institute & $\$ 1,061,000$ & $\$ 0$ & $\$ 1,061,000$ \\
\hline 4 & Tampa & $\begin{array}{l}\text { Increase Federal Research Expenditures and } \\
\text { Economic Impact }\end{array}$ & $\$ 4,872,788$ & $\$ 0$ & $\$ 4,872,788$ \\
\hline 5 & Tampa & $\begin{array}{l}\text { USF/UWF/ Andrews Institute Partnership } \\
\text { Program in Physical Therapy Education, } \\
\text { Research \& Clinical Care }\end{array}$ & $\$ 600,000$ & $\$ 0$ & $\$ 600,000$ \\
\hline 6 & Tampa & $\begin{array}{l}\text { USF Health Neurosciences and Alzheimer's } \\
\text { Disease Initiative }\end{array}$ & $\$ 684,000$ & $\$ 0$ & $\$ 684,000$ \\
\hline 7 & Tampa & Cardiovascular Sciences Initiative & $\$ 412,000$ & $\$ 0$ & $\$ 412,000$ \\
\hline 1 & St. Pete & $\begin{array}{l}\text { Support for Programs in Biology and Middle } \\
\text { Grades STEM Teaching }\end{array}$ & $\$ 549,900$ & $\$ 0$ & $\$ 549,900$ \\
\hline 1 & St. Pete & $\begin{array}{l}\text { Faculty for Programs in Biology and Middle } \\
\text { Grades STEM Teaching }\end{array}$ & $\$ 699,802$ & $\$ 0$ & $\$ 699,802$ \\
\hline 1 & St. Pete & $\begin{array}{l}\text { Staff for Programs in Biology and Middle } \\
\text { Grades STEM Teaching }\end{array}$ & $\$ 359,450$ & $\$ 0$ & $\$ 359,450$ \\
\hline 1 & Sar-Man & Lower-Level Curriculum & $\$ 856,412$ & $\$ 0$ & $\$ 856,412$ \\
\hline 1 & Poly. & $\begin{array}{l}\text { STEM education and engineering; } \\
\text { interdisciplinary with business and innovation } \\
\text { management, and applied research in } \\
\text { Alternative Energy and Biofuels Technologies }\end{array}$ & $\$ 1,632,567$ & $\$ 0$ & $\$ 1,632,567$ \\
\hline & & Total & $\$ 22,993,706$ & $\$ 0$ & $\$ 22,993,706$ \\
\hline
\end{tabular}


University: University of South Florida System

Five-Year Capital Improvement Plan (CIP)

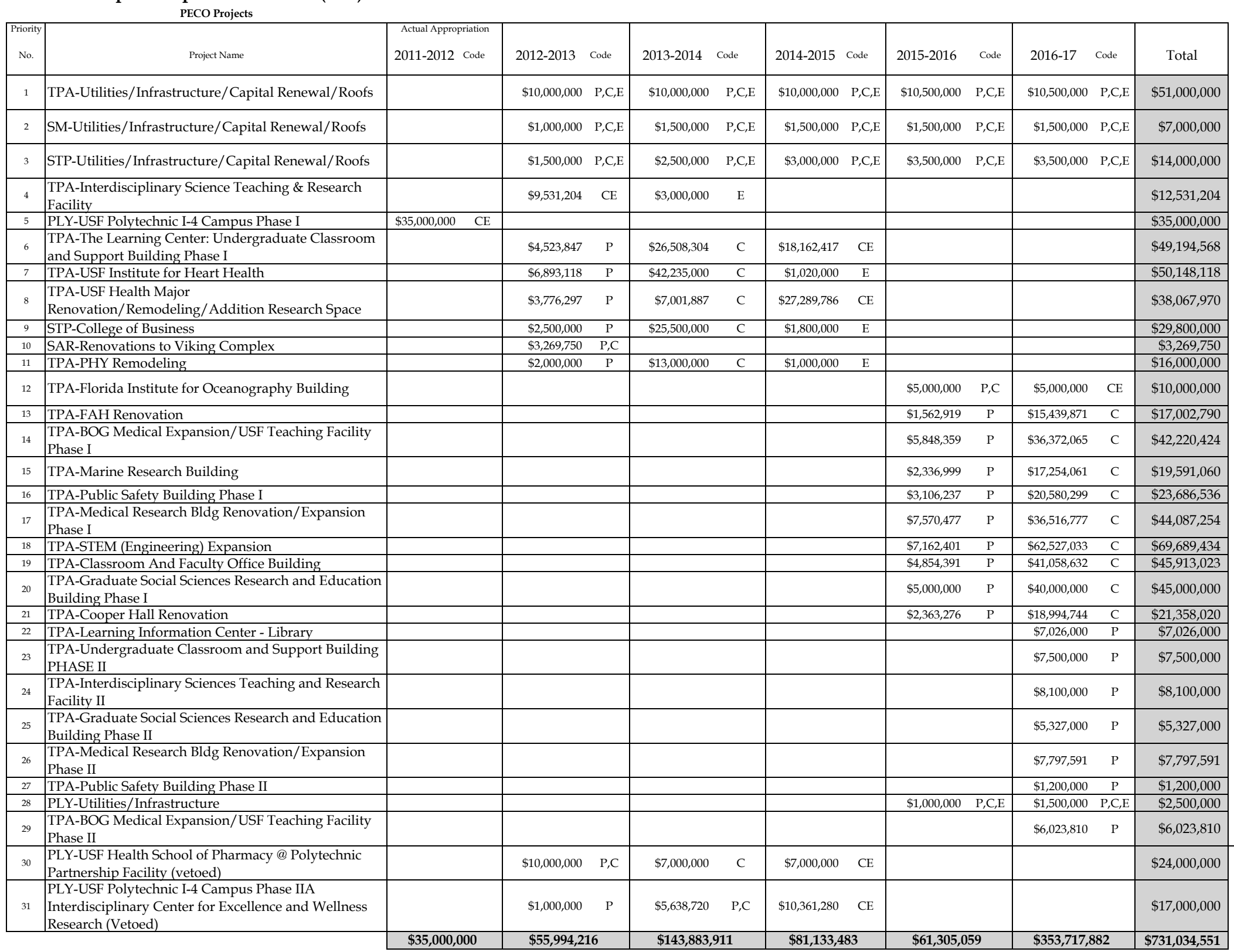

\begin{tabular}{|c|c|c|}
\hline $\begin{array}{c}\text { Educational Plant } \\
\text { Survey } \\
\text { Recommended } \\
\text { (Yes or No) }\end{array}$ & $\begin{array}{c}\text { Academic } \\
\text { Program to } \\
\text { Benefiftrom } \\
\text { Project (e.g., } \\
\text { Biology) }\end{array}$ & $\begin{array}{c}\text { Square Feet } \\
\text { Sres }\end{array}$ \\
\hline Yes & All & \\
\hline Yes & All & \\
\hline Yes & All & \\
\hline Yes & Sciences & 234,549 \\
\hline Yes & Multiple & 117,743 \\
\hline Yes & Multiple & 152,250 \\
\hline Yes & Health & 100,000 \\
\hline Yes & Health & 75,998 \\
\hline Pending & Business & 64,000 \\
\hline Yes & Multiple & 29,347 \\
\hline Yes & Multiple & 81,765 \\
\hline Pending & Marine Science & 22,883 \\
\hline Pending & Fine Arts & 188,452 \\
\hline Yes & Health & 119,100 \\
\hline Pending & Marine Science & 49,409 \\
\hline Yes & All & 72,000 \\
\hline Yes & Health & 95,349 \\
\hline Yes & Multiple & 189,729 \\
\hline Yes & Multiple & 180,000 \\
\hline Yes & Social Sciences & 142,000 \\
\hline Yes & Multiple & 129,896 \\
\hline Pending & All & 103,000 \\
\hline Yes & Multiple & 208,625 \\
\hline Yes & Sciences & 179,999 \\
\hline Yes & Social Sciences & 142,000 \\
\hline Yes & Health & 88,400 \\
\hline Yes & All & 10,500 \\
\hline Yes & All & \\
\hline Yes & Health & 150,000 \\
\hline Yes & Pharmacy & 64,500 \\
\hline Yes & 37,500 \\
\hline
\end{tabular}

Challenge Grant Projects

\begin{tabular}{|c|c|c|c|c|c|c|}
\hline Challenge Grant Projects & $\$ 0$ & $\mathbf{\$ 2 2 , 4 1 0 , 0 6 0}$ & $\mathbf{\$ 0}$ & $\mathbf{\$ 0}$ & $\mathbf{\$ 0}$ & $\mathbf{\$ 0}$ \\
\hline TOTAL & $\mathbf{\$ 2 2 , 4 1 0 , 0 6 0}$ \\
\hline
\end{tabular}


University: University of South Florida System

Five-Year Capital Improvement Plan (CIP)

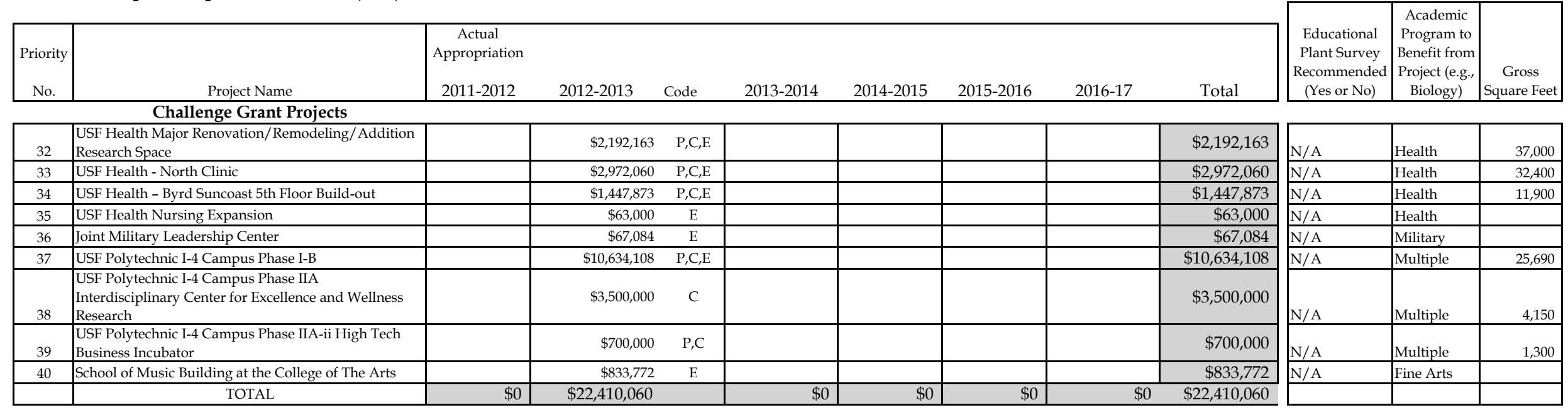

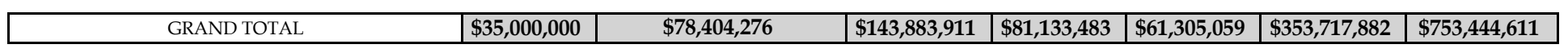

Codes: $\quad \mathrm{P}=$ Planning $\mathrm{C}=$ Construction $\mathrm{CE}=$ Construction $/$ Equipment $\quad \mathrm{LA}=$ Land Acquisition 